\title{
Zeiten zum beruflichen Lernen: Eine empirische Untersuchung zum Zeitpunkt und der Dauer von Fortbildungsangeboten für Lehrkräfte
}

\author{
Eric Richter (ID · Alexandra Marx • Yizhen Huang • Dirk Richter
}

Online publiziert: 28. Januar 2020

(C) Der/die Autor(en) 2020

Zusammenfassung Die Forschung zur Lehrerfortbildung weist darauf hin, dass zeitliche Einschränkungen eine Barriere für die Teilnahme an Fortbildungen darstellen. Insbesondere die Unvereinbarkeit der Arbeitszeit mit dem Zeitpunkt des Fortbildungsangebotes wird von Lehrkräften als hinderlich wahrgenommen. Welche zeitlichen Merkmale das Fortbildungsangebot für Lehrkräfte aufweist und ob diese im Zusammenhang mit der Fortbildungsteilnahme von Lehrkräften stehen, fand bisher wenig Berücksichtigung. Die vorliegende Studie verfolgt daher das Ziel, zeitliche Merkmale des Fortbildungsangebotes für Lehrkräfte zu beschreiben und ihre Vorhersagekraft für die Fortbildungsteilnahme zu untersuchen. Die Daten hierfür stammen aus der elektronischen Datenbank für Lehrerfortbildungen des Landes Brandenburg im akademischen Jahr 2016/2017. Der Datensatz umfasst 1330 schulexterne Lehrerfortbildungen staatlicher Anbieter. Die Ergebnisse zeigen, dass Dauer und Zeitpunkt einer Fortbildungsveranstaltung prädiktiv für die Teilnahme der Lehrkräfte sind. Die Befunde erweitern damit den Diskurs zu Bedingungen des beruflichen Lernens von Lehrkräften und liefern Implikationen für eine passgenaue Fortbildungsplanung.

\footnotetext{
E. Richter $(\varangle) \cdot$ Dr. Y. Huang $\cdot$ Prof. Dr. D. Richter

Department Erziehungswissenschaft, Universität Potsdam, Karl-Liebknecht-Straße

24-25, 14476 Potsdam, Deutschland

E-Mail: eric.richter@uni-potsdam.de

Dr. Y. Huang

E-Mail: yihuang@uni-potsdam.de

Prof. Dr. D. Richter

E-Mail: dirk.richter@uni-potsdam.de

Dr. A. Marx

Die Deutsche Schulakademie, Hausvogteiplatz 12, 10117 Berlin, Deutschland

E-Mail: alexandra.marx@deutsche-schulakademie.de
} 
Schlüsselwörter Berufliches Lernen · Lehrerbildung · Lehrerfortbildung · Lernzeit · Polynomial Regression

\title{
Time for professional learning: An empirical study about timing and duration of teacher training
}

\begin{abstract}
Research on teachers' professional development suggests that time constraints often prevent teachers from participating in in-service trainings. In particular, a time conflict between work and in-service training seems to be an obstacle for many teachers. However, little empirical evidence exists that investigates effects of temporal variables such as timing and duration of the training session on teachers' participation rate. The present study aims to describe time and duration of professional development activities and its relationship with the number of attendees in the respective courses. The data of the study were provided from the electronic database on teachers' professional development activities in the state of Brandenburg during the academic year 2016-2017. The data set comprises 1330 professional development activities provided by state agencies. The results show that both time and duration significantly predict the participation rate in the training courses. The findings broaden the scope of the discussion about conditions of teachers' professional learning and provide implications for a well-fitting in-service training schedule.
\end{abstract}

Keywords Continuing education - Learning time $\cdot$ Polynomial regression · Professional development $\cdot$ Teacher training

\section{Einleitung}

Die Teilnahme an Lehrerfortbildungen stellt einen wichtigen Beitrag zur Entwicklung der professionellen Kompetenz vor Lehrkräften dar (Lipowsky 2014). Die Untersuchung der Nutzung dieser Angebote bildet entsprechend einen zentralen Gegenstand der Lehrkräfteforschung (Richter 2016). Dabei wurde bisher vor allem untersucht, in welchen Umfängen Fortbildungsangebote in Anspruch genommen werden (z.B. Hoffmann und Richter 2016) und welche Lehrkräfte Fortbildungen nutzen (z. B. Richter et al. 2011). Bislang unbeachtet blieb die Frage, welche Fortbildungsangebote Lehrkräften überhaupt unterbreitet werden - und ob Merkmale dieser Angebote im Zusammenhang mit dem Teilnahmeverhalten von Lehrkräften stehen. Bei der Frage des Angebots spielen einerseits die bereitgestellten Veranstaltungsinhalte eine wichtige Rolle, aber andererseits auch die Zeiten, zu denen die Fortbildungen stattfinden. Die zeitlichen Merkmale von Lehrerfortbildungen sind insbesondere deshalb bedeutsam, da Lehrkräften in der Regel keine festen Lernzeiten zur Verfügung gestellt werden und sie dennoch ihren Beruf mit ihrer professionellen Weiterentwicklung verbinden müssen. Tatsächlich berichten Lehrkräfte in nationalen Large Scale Studien, dass fehlende zeitliche Ressourcen einen Hauptgrund für die Nicht-Teilnahme an Fortbildungen darstellen (Richter et al. 2012, 2018) und dies obwohl erwartet wird, eher die unterrichtsfreie Zeit für Fortbildungen zu nut- 
zen (DVLfB 2018). Die Frag, welche Zeiten Lehrkräfte letztlich zum Lernen nutzen, kann auf Grundlage bisheriger Untersuchungen nicht beantwortet werden.

Eine Annäherung an diese Forschungslücke ermöglichen Angebotsanalysen von Lehrerfortbildungen auf der Grundlage von Datenbankauswertungen (Nolda 2009). Erste Untersuchungen zum Angebot von Lehrerfortbildungen nahmen beispielsweise in den Blick, inwiefern sich bildungspolitische Reformen im Fortbildungsangebot widerspiegeln (Cramer et al. 2019). Ob ein entsprechendes Angebot jedoch auch von den adressierten Lehrkräften genutzt wurde, war bisher ebenso wenig Bestandteil von Untersuchungen wie andere Angebotsmerkmale. Die vorliegende Untersuchung erweitert vor diesem Hintergrund diesen in der Forschung zur Lehrerfortbildung bisher wenig genutzten Ansatz der Angebotsanalysen und untersucht am Beispiel des Landes Brandenburg zum einen die dort angebotenen Veranstaltungen des akademischen Jahres 2016/2017 hinsichtlich zeitlicher Merkmale. Hierbei konzentrieren wir uns auf die Dauer der Fortbildungsangebote sowie den Zeitpunkt, zu dem ein bestimmtes Angebot gemacht wird. Zum anderen soll empirisch überprüft werden, ob zwischen diesen zeitlichen Angebotsmerkmalen und der Fortbildungsnutzung systematische Zusammenhänge bestehen. Die Identifizierung etwaiger Zusammenhänge könnte dabei Hinweise für eine passgenaue Fortbildungsplanung und Bedarfsermittlung liefern und den wissenschaftlichen Diskurs zu den Bedingungen des beruflichen Lernens von Lehrkräften um eine strukturelle Ebene erweitern.

Im Folgenden wird zunächst die Teilnahme von Lehrkräften an Maßnahmen zur professionellen Entwicklung beschrieben und mit Hilfe eines allgemeinen Modells zur Beschreibung von Fortbildungsbeteiligung in der Erwachsenenbildung (Boeren et al. 2010) theoretisch verortetet. Zeitliche Angebotsmerkmale (Dauer, Zeitpunkt) werden dabei als mögliche bedeutsame Konstrukte zur Erklärung von Fortbildungsbeteiligung identifiziert und anschließend vor dem Hintergrund bestehender Befunde der allgemeinen Weiterbildungsforschung diskutiert.

\subsection{Teilnahme von Lehrkräften an Maßnahmen zur professionellen Entwicklung}

Die empirische Untersuchung der Teilnahme von Lehrkräften an Fortbildungen stellt einen zentralen Bestandteil der Lehrkräfteforschung dar. Lehrerfortbildungen werden hierbei als formale berufliche Lerngelegenheiten für praktizierende Lehrkräfte verstanden, die der Aktualisierung und Vertiefung von Wissen und Kompetenzen dienen, die im Rahmen des Studiums und des Vorbereitungsdienstes erworben wurden. Lehrkräftefortbildungen repräsentieren einen zentralen Teil beruflicher Lerngelegenheiten in der sogenannten Dritten Phase der Lehrerbildung. Sie lassen sich konzeptuell von non-formalen (z.B. professionelle Lerngemeinschaften) und informellen Lerngelegenheiten (z. B. Austausch von Materialien) unterscheiden (Richter 2016). Trotz der Vielfalt möglicher Lerngelegenheiten für das berufliche Lernen von Lehrkräften sollen im Folgenden wegen ihres besonderen Stellenwertes für die berufliche Weiterentwicklung von Lehrkräften ausschließlich Fortbildungen adressiert werden. Ihre von verschiedenen Autor/innen postulierte Bedeutung ergibt sich dabei aus ihren spezifischen Charakteristika (z. B. Lipowsky 2014; Richter 2016; DarlingHammond et al. 2017). So handelt es sich bei Lehrerfortbildungen um pädagogisch 
und didaktisch vorstrukturierte Lernangebote, die in der Regel durch fachliche Expert/innen unterbreitet werden. Sie ermöglichen grundsätzlich das gemeinsame Lernen von Lehrkräften in vergleichsweise kurzen Zeiträumen und stellen somit eine potenzielle Bereicherung für die berufliche Praxis von Lehrkräften dar. Entsprechend der hohen Bedeutung von Lehrerfortbildungen unterhalten alle Bundesländer unter Einsatz hoher finanzieller Mittel Infrastrukturen für staatliche Lehrerfortbildungen, die es prinzipiell allen Lehrkräften ermöglichen, kostenfrei und den eigenen Bedürfnissen entsprechend diese Lerngelegenheiten wahrzunehmen (DVLfB 2018).

Die Teilnahme an solchen Fortbildungsveranstaltungen ist seit längerer Zeit im Fokus der empirischen Bildungsforschung. Der Fokus der Fortbildungsforschung reicht dabei von der reinen Erfassung der Teilnahmequoten bis hin zur Ergründung des individuellen Teilnahmeverhaltens sowie den dahinterliegenden Determinanten (Richter 2016). Als Ergebnis verschiedener Lehrkräftebefragungen lässt sich zunächst festhalten, dass ca. $80 \%$ der Lehrkräfte in Deutschland regelmäßig an Fortbildungen teilnehmen (Hoffmann und Richter 2016). Dieser Befund zeigt sich über verschiedene Fächer hinweg sowohl für Lehrkräfte an Grundschulen (Richter et al. 2012) als auch für Lehrkräfte an weiterführenden Schulen (Richter et al. 2013; Hoffmann und Richter 2016). Lehrkräfte, die regelmäßig an Fortbildungen teilnehmen, unterscheiden sich dabei erheblich in der Zeit, die sie in Fortbildungen investieren. So konnte beispielsweise in einer Lehrkräftebefragung des IQB-Ländervergleichs 2012 gezeigt werden, dass $29 \%$ der Lehrkräfte innerhalb von zwei Schuljahren nur maximal an zwei Fortbildungen teilnehmen, während $25 \%$ der Lehrkräfte mindestens sechs Fortbildungsveranstaltungen aufsuchen (Richter et al. 2013). Ähnliche Befunde berichten Wei et al. (2010) auch für US-amerikanische Lehrkräfte. Die vorgestellten Forschungsbefunde machen demnach deutlich, dass sich Lehrkräfte in der Häufigkeit der Nutzung von Fortbildungen stark voneinander unterscheiden.

Zur Erklärung der unterschiedlich starken Nutzung beruflicher Lerngelegenheiten durch erwachsene Lerner haben sich in der Fort- und Weiterbildungsforschung zunehmend Mehrebenenmodelle etabliert (Boeren et al. 2010). Diese heuristischen Modelle unterscheiden zunächst zwischen einer Nachfrage- und einer Angebotsseite, wobei zwischen beiden Seiten eine interdependente Beziehung angenommen wird. Baert et al. (2006) umschreiben diese Beziehung als einen Kreislauf „from educational need to educational participation“ (S. 95) und meinen damit, dass nicht allein das Lernbedürfnis eines potentiellen Lerners zur Teilnahme an einer Bildungsveranstaltung führt. Ausschlaggebend ist vielmehr, ob und wie das Bildungsangebot durch den potentiellen Lerner wahrgenommen wird.

Derartige Nachfrage-Angebotsmodelle gehen ferner von der Annahme aus, dass es neben einer Nachfrage- und Angebotsseite unterschiedliche Ebenen gibt, die auf den Prozess der Entscheidungsfindung wirken. Entscheidender Akteur auf der Nachfrageseite ist dabei das Individuum (z.B. Lehrkraft), welches auf der sogenannten Mikroebene agiert und durch psychologische, sozioökonomische sowie kulturelle Faktoren, aber auch durch relevante Andere (z. B. Familie, Bezugsgruppe) beeinflusst wird. Das Handeln des Individuums ist ferner umrahmt durch Regeln, Gesetze und Verpflichtungen (z.B. Schulgesetz), welche als Ausdruck eines generellen gesellschaftlichen Kontextes verstanden werden können und die sogenannte Makroebene repräsentieren (Boeren et al. 2010; Abb. 1). 


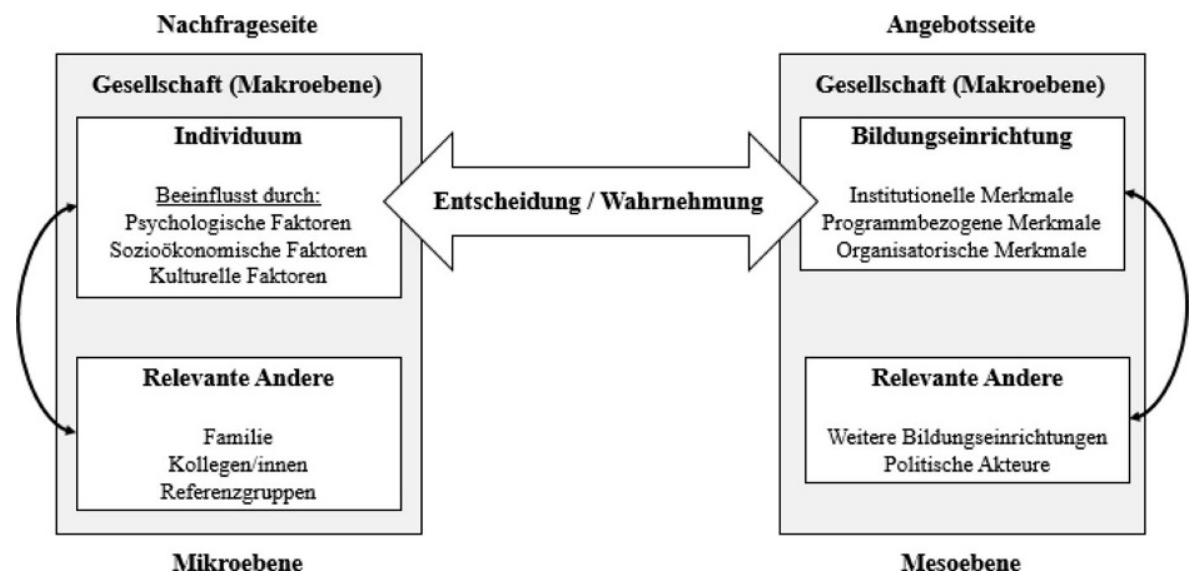

Abb. 1 Mehrebenen-Nachfrage-Angebotsmodell zur Erklärung des beruflichen Lernens von Lehrpersonen. (Nach Boeren et al. 2010, S. 56)

Den Kern der Angebotsseite stellen Bildungseinrichtungen dar, welche auf der sogenannten Mesoebene verortet werden können. Diese zeichnen sich durch verschiedene institutionelle Merkmale aus (z. B. Zahl der Mitarbeiter/innen) und weisen ferner spezifische programmbezogene (z. B. Inhalte) sowie organisatorische Merkmale (z.B. Zeitpunkt und Dauer der Veranstaltung) auf. Die konkrete Ausgestaltung einer Bildungseinrichtung wird dabei auf der einen Seite von relevanten Anderen (z.B. politischen Akteuren) beeinflusst. Auf der anderen Seite wird der Handlungsrahmen einer Bildungseinrichtung durch gesellschaftliche Kontextbedingungen (Makroebene) definiert (Boeren et al. 2010). Vor diesem theoretischen Hintergrund kann die Teilnahme erwachsener Lerner an beruflichen Fort- und Weiterbildungsangeboten als „successful interaction between the individual and the educational institutions“ (Boeren et al. 2010, S. 59) verstanden werden.

Im Kontext von Lehrerfortbildungen lässt sich innerhalb Deutschlands eine Vielzahl verschiedener privater und staatlicher Bildungseinrichtungen identifizieren (z.B. Schulämter, private Stiftungen, Universitäten; Altrichter 2010; Richter 2016). Insgesamt nehmen staatliche Anbieter gemäß KMK eine besondere Bedeutung in der Lehrkräftefortbildung ein (KMK 2000), welche sich nicht zuletzt in den hohen finanziellen Aufwendungen der Bundesländer für entsprechende Fortbildungsangebote manifestiert (DVLfB 2018). Ihre Angebote lassen sich grundsätzlich in schulexterne und schulinterne Angebote unterscheiden (Richter 2016). Aufgrund der Tatsache, dass externe Angebote allen Lehrkräften eines Landes offenstehen und somit von diesen angewählt werden können, steht diese Gruppe von Fortbildungsangeboten im Fokus der vorliegenden Arbeit.

Während bereits eine Reihe von Arbeiten existiert, die versuchen, das Teilnahmeverhalten an Lehrerfortbildungen empirisch durch persönliche Voraussetzungen (z.B. Teilnahmemotivation, siehe Rzejak et al. 2014; Gorozidis und Papaioannou 2014) oder kontextuale Bedingungen (z. B. Fortbildungswertigkeit im Kollegium, siehe Richter et al. 2010) zu erklären, liegen empirische Studien zum Zusammenhang zwischen verschiedenen Merkmalen des Fortbildungsangebots, wie etwa dem 
Zeitpunkt, der Dauer oder dem Inhalt und dem Teilnahmeverhalten von Lehrkräften bislang kaum vor. Der Einfluss solcher Merkmale auf die Teilnahme an Fortbildungen wurde bisher vorrangig indirekt durch die Ergründung von Teilnahmebarrieren abgeschätzt.

Über verschiedene Erhebungen hinweg zeigte sich dabei, dass insbesondere zeitliche Merkmale des Fortbildungsangebotes bedeutsam für die Entscheidung für oder gegen die Teilnahme sein könnten. So beschreiben Lehrkräfte der Sekundarstufe I die Unvereinbarkeit der Arbeitszeit mit dem Fortbildungsangebot als besonders hinderlich für die Teilnahme an Fortbildungen (Richter et al. 2018). Dieser Befund deckt sich mit Ergebnissen einer Befragung von Grundschullehrkräften, die als subjektive Hinderungsgründe für die Teilnahme an Fortbildungen ebenfalls fehlende zeitliche Ressourcen in Folge hoher beruflicher Belastungen nennen (Richter et al. 2012). Ähnliche Befunde liefern ältere nationale sowie internationale Untersuchungen, die ebenfalls zeigen konnten, dass Zeitmangel eine zentrale Lernbarriere darstellt (Collinson und Fedoruk Cook 2001), die sich aus der eigenen Unterrichtsverpflichtung (Graudenz et al. 1995; Beck und Ullrich 1996), der Unbeweglichkeit schulischer Termine und einer als generell hoch eingeschätzten Arbeitsbelastung (Wolf et al. 1997, 1999) ergibt. Während Teilnahmebarrieren, die mit Merkmalen von Lehrkräften verbunden sind, bereits in Ansätzen gut erforscht wurden, so bestehen für Teilnahmebarrieren, die mit den Merkmalen des Angebots zusammenhängen, bisher kaum empirische Befunde. Erste Studien zu diesem Thema zeigen, dass auch ungünstige Veranstaltungstermine aus Sicht von Lehrkräften Barrieren für die Teilnahme an Fortbildungen darstellen (Wolf et al. 1997).

Die Frage, inwieweit zeitliche Merkmale des Fortbildungsangebotes tatsächlich erklärungsmächtig für die Teilnahme an Fortbildungen sind, kann auf Grundlage der bisherigen Forschung nicht beantwortet werden. Um sich der Beantwortung der Frage zu nähern, soll deshalb im folgenden Kapitel die Bedeutung der Zeit zum beruflichen Lernen von Lehrkräften herausgearbeitet werden. Ausgehend davon wird anhand verschiedener Rechtsvorschriften die aktuelle Situation zur Lernzeit von Lehrkräften in Deutschland dargestellt. Vor diesem Hintergrund werden empirische Befunde, die sich mit der Frage auseinandersetzen, wann Erwachsene lernen, diskutiert.

\subsection{Zeiten zum Lernen}

Die Teilnahme an beruflichen Lerngelegenheiten durch Erwachsene kann als Ausdruck einer gelungenen Interaktion zwischen der Nachfrage des Individuum und dem Angebot einer Bildungseinrichtung verstanden werden (Boeren et al. 2010). Aus der allgemeinen Arbeits- und Organisationspsychologie ist diesbezüglich bekannt, dass u. a. das Vorhandensein zeitlicher Restriktionen diesen Prozess erschweren kann (Scheffer und Kuhl 2010; Schaper 2019). Befunde zum Fortbildungsverhalten verschiedener Berufsgruppen, wie z.B. Ärzte, deuten vor diesem Hintergrund darauf hin, dass zeitliche Aspekte im Zusammenhang mit der Nutzung beruflicher Lerngelegenheiten zu stehen scheinen (Gerlach und Beyer 1999; Heintze et al. 2005). Die Bedeutung zeitlicher Merkmale von Lerngelegenheiten für das Lernen von Lehrkräften stand jedoch bisher nur bedingt im Fokus der Forschung. Allgemein lässt sich 
diesbezüglich zunächst festhalten, dass die Bereitstellung von Lernzeit ein wichtiger Faktor für die Realisierung von schulischen und außerschulischen Bildungs- und Veränderungsprozessen ist (Schmidt-Lauff 2009). Das Vorhandensein ausreichender Lernzeit ist vor diesem Hintergrund auch für nachhaltiges berufliches Lernen von Lehrkräften grundlegend (Yoon et al. 2007; Desimone und Garet 2015; DarlingHammond et al. 2017). Entsprechend gab es bereits in den 90er Jahren einen Aufruf des US Department of Education, Lehrkräfte substanziell mit zeitlichen Ressourcen für das berufliche Lernen auszustatten (,Creating time for professional development. Teachers need regular, scheduled blocks of time for working and learning together. It could be considered legitimate for teachers to spend time away from students and performing these activities“, nach: Loucks-Horsley et al. 1996, S. 5; vgl. US Department of Education 1995). Dieser Appell scheint sich jedoch bisher weder in der Realität der Lehrkräfte noch in den hiesigen Schulgesetzen niedergeschlagen zu haben.

So zeigen Studien, dass Lehrkräfte insgesamt nur einen geringen Teil ihrer Arbeitszeit für ihre berufliche Fortbildung aufwenden. In verschiedenen nationalen wie internationalen Untersuchen zum Zeitbudget von Lehrkräften berichten Lehrkräfte über hohe durchschnittliche Wochenarbeitszeiten von $45,8 \mathrm{~h}$ bis hin $\mathrm{zu}$ mehr als 50 h (Bruno et al. 2012; Philipp und Kunter 2013; Richter und Pant 2016). In einer Untersuchung zum Verlauf der Wochenarbeitszeiten von Lehrkräften konnte jedoch gezeigt werden, dass sich diese über ein Schuljahr hinweg ungleich verteilen und saisonale Schwankungen zu verzeichnen sind (Mußmann et al. 2016). Typische Belastungsspitzen finden sich über verschiedene Schulformen hinweg jeweils vor den Zeugnissen, wenn Lehrkräfte zusätzlich zur regulär anfallenden Arbeit Noten eintragen, Zeugnisse und Gutachten anfertigen und an Zensurenkonferenzen teilnehmen müssen. Sehr geringe Arbeitszeiten berichten Lehrkräfte dagegen erwartungsgemäß während der Schulferien (Mußmann et al. 2016). Obwohl Lehrkräfte somit über hohe durchschnittliche Wochenarbeitszeiten berichten, verwenden sie nur einen sehr geringen Teil dieser Zeit für berufliches Lernen (ca. $2 \mathrm{~h}$, siehe: Philipp und Kunter 2013). Diese Befundlage überrascht insofern nicht, als dass die kontinuierliche Fortbildung für Lehrkräfte in allen Bundesländern per Schulgesetz zwar prinzipiell verpflichtend ist (DVLfB 2018; für Brandenburg s. BbgSchulG 2002), in der Regel jedoch keine individuellen oder kollektiven Lernzeitfenster vorgehalten werden. Stattdessen existieren in einer Reihe von Rechtsvorschriften Ausführungshinweise zur Fortbildungsteilnahme in den einzelnen Ländern. Diese schreiben vor, dass die Teilnahme an Fortbildungen grundsätzlich genehmigungspflichtig ${ }^{1}$ ist und entsprechend vorab in der Regel bei der Schulleitung beantragt werden muss. Die Bewilligung der Teilnahme hängt dann unter anderem davon ab, ob ihr etwaige dienstliche Gründe, z. B. der Ausfall von Unterricht, entgegenstehen. Werden seitens der Vorgesetzten dienstliche Gründe geltend gemacht, kann die Bewilligung der Fortbildungsteilnahme verwehrt werden. Einige Länder (z. B. Hamburg) gewähren darüber hinaus grundsätzlich überhaupt keinen Anspruch auf Freistellungen während der Un-

\footnotetext{
1 Ausgenommen davon ist die Abordnung zur Teilnahme an Veranstaltungen, die im ausschließlich dienstlichen Interesse stattfindet. Ein ausschließlich dienstliches Interesse liegt etwa dann vor, wenn eine Fortbildungsteilnahme durch die Dienstvorgesetzte/den Dienstvorgesetzen angeordnet wird.
} 
terrichtszeit und verweisen auf die Nutzung der unterrichtsfreien Zeit (DVLfB 2018). Fortbildungen werden vor diesem Hintergrund in den meisten Bundesländern vorrangig halbtägig, außerhalb der regulären Unterrichtszeit angeboten (Amrhein und Badstieber 2015; Bayerisches Staatsministerium für Bildung 2017; DVLfB 2018). Eine aktuelle Untersuchung zum Fortbildungsangebot in Baden-Württemberg untermauert dies, indem sie zeigt, dass ca. $75 \%$ aller Fortbildungsangebote lediglich einen halben Tag umfassen (Cramer et al. 2019). Somit besteht für viele Lehrkräfte nur eine eingeschränkte Möglichkeit, Fortbildungen über einen längeren Zeitraum bzw. während der Unterrichtszeit zu besuchen.

Der Umstand, dass für Lehrkräfte in der Regel keine festen Lernzeiten zur Verfügung gestellt werden, wirft die Frage auf, wann Lehrkräfte formale Lerngelegenheiten in Anspruch nehmen und wie viel Zeit sie sich dafür nehmen. Eine erste Annäherung ermöglichen Untersuchungen von Erwartungen und Wünschen an Fortbildungen aus Sicht von Lehrkräften. Hinsichtlich der Dauer von Fortbildungsveranstaltungen zeigte sich, dass sich Lehrkräfte eher ganztägige und somit zeitlich längere Fortbildungsangebote zwischen 6 und 8h wünschen (Daus et al. 2004; Jäger und Bodensohn 2007). Als mögliche Erklärung hierfür führen Kanwischer et al. (2004) die höhere Passung zum Arbeitsrhythmus der Lehrkräfte an, da Lehrkräfte im Falle ganztägiger Fortbildungen keinen eigenen Unterricht halten müssen und sich aus diesem Grund stärker auf den eigentlichen Gegenstand der Fortbildung einlassen können. Halbtägige Fortbildungen fänden dagegen verstärkt nach der regulären Unterrichtszeit statt. Sie würden deshalb als Zusatzbelastung verstanden und kollidierten darüber hinaus potentiell mit familiären Verpflichtungen (Kanwischer et al. 2004). Dabei wäre ein Großteil der Lehrkräfte auch bereit, mehrtägige Veranstaltungen zu besuchen (Jäger und Bodensohn 2007) oder die unterrichtsfreie Zeit während der Schulferien für Fortbildungen zu nutzen (Daus et al. 2004).

Eine stärker empirische Annäherung an die Fortbildungsnutzung ermöglichen Befunde zu Lernzeiten in der Erwachsenenbildung. In der einschlägigen Forschungsliteratur wird allgemein betont, dass es bei den Lernzeiten Erwachsener große zeitliche Konkurrenzfaktoren, wie Arbeits- und Familienzeit gibt (Schmidt-Lauff 2008; Lobe 2015). Beide besitzen einen erheblichen Einfluss sowohl auf die Verteilung der Lernzeiten (Zeitpunkt) als auch auf das Ausmaß der Lernzeiten (Dauer).

Hinsichtlich des Ausmaßes von Lernzeiten wird davon ausgegangen, dass Weiterbildungsangebote umso schwieriger mit anderen Lebensbereichen zu vereinbaren sind, je mehr zeitliche Ressourcen diese Angebote beanspruchen (Lobe 2015). Vor diesem Hintergrund konnte im Bereich der Erwachsenenbildung gezeigt werden, dass zeitlich kompaktere Termine gegenüber zeitintensiveren Angeboten priorisiert werden, da diese einen weniger starken Einschnitt in das private und/oder berufliche Leben darstellen (Denninger et al. 2017). Dass diese Befunde auch für Lehrkräfte relevant sein können, verdeutlichen zum Teil die bereits angeführten Untersuchungen zu den Teilnahmebarrieren an Lehrerfortbildungen, in denen gezeigt werden konnte, dass Nicht-Teilnehmer/innen ihre beruflichen Verpflichtungen als zentralen Hinderungsgrund für die Nicht-Teilnahme an Lehrerfortbildungen wahrnehmen (Richter et al. 2018).

Neben der Frage nach der Dauer liegen auch erste Befunde zur Frage vor, zu welchem Zeitpunkt sich Erwachsene fortbilden. Einen bedeutsamen Einflussfaktor 
stellt hierbei die subjektive Bewertung der Qualität von Lernzeit dar, welche sich auf die Wertigkeit bezieht, die ein Individuum einer bestimmten Tageszeit zuschreibt (Schmidt-Lauff 2010). Den Hintergrund hierfür bilden u. a. Aspekte der physischen und emotionalen Befindlichkeit: Lernen ist demnach zum Beispiel an die eigene körperliche Verfassung bzw. die selbsteingeschätzte Aufnahmefähigkeit geknüpft. Dem Vormittag wird dabei von Weiterbildungsteilnehmer/innen eine hohe subjektive Aufnahmefähigkeit - und somit eine hohe Zeitqualität - zugeschrieben (Schmidt-Lauff 2008, 2009). Da auf der anderen Seite auch Aktivitäten, die vor respektive nach einem Lernangebot stattfinden, die individuell empfundene Zeitqualität beeinflussen können (Nahrstedt und Brinkmann 1998), werden abendliche Veranstaltungen nach Erledigung aller beruflicher und familiärer Pflichten - eher als anstrengender eingeschätzt (Schmidt-Lauff 2008, 2009). Zu ähnlichen Befunden kommt Präßler (2015) für die wissenschaftliche Weiterbildung. Demgegenüber stehen jedoch Befunde aus der Zeitverwendungserhebung des Statistischen Bundesamtes (2015), die darauf hindeuten, dass die durchschnittliche Zeitverwendung für Weiterbildung innerhalb und außerhalb der Arbeitszeit ähnlich verteilt ist. Diese Ergebnisse deuten somit nicht darauf hin, dass Angebote am Vormittag bevorzugt aufgesucht werden. Kritisch angemerkt sei jedoch, dass bei diesem Befund nicht zwischen verschiedenen Personen- und Altersgruppen unterschieden wurde. Letztlich existieren sowohl für Angebote am Vormittag (berufliche Verpflichtung) als auch für Angebote am Nachmittag (familiäre und private Verpflichtung) bedeutsame konkurrierende Verpflichtungen (Denninger et al. 2017).

Zusammenfassend lässt sich festhalten, dass die Bedeutung zeitlicher Merkmale von Lehrerfortbildungen, wie beispielsweise dem Zeitpunkt oder der Dauer einer konkreten Veranstaltung, für das Teilnahmeverhalten von Lehrkräften bisher nur unzureichend erforscht wurde. Gleichwohl lässt sich unter Berücksichtigung der Befunde der allgemeinen Weiterbildungsforschung annehmen, dass die zeitlichen Merkmale einer Veranstaltung mit dem Teilnahmeverhalten von Personen zusammenhängen könnten. Die Frage, welche Zeiten Lehrkräfte zum beruflichen Lernen nutzen, kann auf Grundlage der bisherigen Forschung nicht beantwortet werden. Die vorliegende Untersuchung versucht, diese Forschungslücke zu schließen, indem die zeitlichen Merkmale des Fortbildungsangebotes zunächst analysiert und in einem zweiten Schritt auf etwaige Zusammenhänge mit den Teilnahmequoten geprüft werden.

\subsection{Fragestellung und Hypothesen}

Die vorliegende Arbeit verfolgt zwei übergeordnete Ziele. Sie versucht erstens das Fortbildungsangebot für Lehrkräfte auf Grundlage der zeitlichen Fortbildungsmerkmale Dauer und Zeitpunkt der angebotenen Fortbildungsveranstaltungen zu beschreiben. Zweitens versucht sie, Zusammenhänge zwischen diesen zeitlichen Merkmalen des Fortbildungsangebotes auf der einen Seite und Teilnehmerzahlen von Fortbildungsveranstaltungen auf der anderen Seite zu ergründen. Entsprechende Zusammenhänge können sowohl theoretisch, auf Grundlage des vorgestellten Nachfrage-Angebotsmodells (Boeren et al. 2010), als auch im Hinblick auf empirische Befunde der allgemeinen Weiterbildungsforschung angenommen werden (Schmidt- 
Lauff 2008, 2009; Denninger et al. 2017). Hieraus lassen sich drei Forschungsfragen ableiten:

\subsubsection{Forschungsfrage 1}

Wie lange dauern Fortbildungsveranstaltungen für Lehrkräfte und bestehen Zusammenhänge zwischen der Dauer und der Anzahl der Teilnehmer/innen?

Hypothese 1 Lehrkräfte in Deutschland berichten über hohe Wochenarbeitsstunden (Philipp und Kunter 2013; Richter und Pant 2016) und verfügen über keine institutionalisierten Lernzeitfenster (DVLfB 2018). Auf Grund der hohen zeitlichen Einschränkungen, die sich daraus ergeben, wird angenommen, dass Lehrkräfte eher kürzere als längere Fortbildungsveranstaltungen besuchen. Diese Annahme wird zusätzlich durch Befunde der allgemeinen Weiterbildungsforschung gestützt, die darauf hindeuten, dass kürzere Lerngelegenheiten weniger stark in Konkurrenz zu anderen Lebenszeiten stehen und aus diesem Grund bevorzugt genutzt werden (Lobe 2015; Denninger et al. 2017).

\subsubsection{Forschungsfrage $2 a$}

$\mathrm{Zu}$ welcher Uhrzeit beginnen Fortbildungsveranstaltungen für Lehrkräfte und hängt der Zeitpunkt des Beginns mit der Anzahl der Teilnehmer/innen zusammen?

Hypothese 2 Die Teilnahme an Lehrerfortbildungen für Lehrkräfte unterliegt der Genehmigungspflicht in der Regel durch die Schulleitung. Diese Genehmigung kann verwehrt werden, wenn dienstliche Gründe, wie beispielsweise unterrichtliche Verpflichtungen, dagegensprechen. Generell gilt, dass Lehrkräfte angehalten sind, die unterrichtsfreie Zeit für Fortbildungsveranstaltungen zu nutzen (DVLfB 2018). Es wird deshalb angenommen, dass Lehrkräfte hinsichtlich der Tageszeit einer Fortbildungsveranstaltung eher Angebote außerhalb der Unterrichtszeit, also eher am Nachmittag, aufsuchen.

\subsubsection{Forschungsfrage $2 b$}

Zu welchen Zeitpunkten im Schuljahr werden Fortbildungsveranstaltungen für Lehrkräfte angeboten und hängt dieser Zeitpunkt mit der Anzahl der Teilnehmer/innen zusammen?

Hypothese 3 Die Arbeitsbelastung für Lehrkräfte innerhalb eines Schuljahres schwankt und insbesondere vor der Vergabe der Halbjahres- und Jahreszeugnisse sind saisonale Belastungsspitzen zu verzeichnen (Mußmann et al. 2016). Es wird angenommen, dass das Ausmaß der beruflichen Eingebundenheit mit dem Teilnahmeverhalten der Lehrkräfte zusammenhängt (Richter et al. 2018) und die Zahl der Fortbildungsteilnehmer/innen im Verlauf eines Schuljahres schwankt. Insbesondere in Monaten, von denen angenommen werden kann, dass sie wegen der anstehenden 
Vergabe der Zeugnisse sehr arbeitsintensiv sind (Januar, Juni), wird eine geringere Fortbildungsbeteiligung erwartet.

\section{Methoden}

Grundlage für die vorliegende Untersuchung sind die in einer elektronischen Datenbank hinterlegten Fortbildungsangebotsdaten für das Land Brandenburg aus dem akademischen Jahr 2016/2017 (01.08.2016 bis 31.07.2017). Das Land Brandenburg verwendet für die Verwaltung der Lehrerfortbildungen des Landes die sogenannte TIS-Datenbank, in welcher die Angebote erfasst und die Teilnahmen der Lehrkräfte dokumentiert werden. Über einen Online-Zugang bietet das TIS-Portal die Möglichkeit der dezentralen Fort- und Weiterbildungsplanung durch verschiedene staatliche Gruppen und Anbieter im Bildungsmanagement. Angebote von nichtstaatlichen Anbietern, wie etwa Verlagen, befinden sich nicht in der TIS-Datenbank und können aus diesem Grund nicht in den Analysen berücksichtigt werden. ${ }^{2}$

Bevor die Datengrundlage im Folgenden ausführlich beschrieben wird, soll zunächst kurz das System der staatlichen Lehrkräftefortbildung in Brandenburg vorgestellt werden. Wie in allen Bundesländern, haben auch Lehrkräfte in Brandenburg das Recht und die Pflicht, ihre Kenntnisse und Fähigkeiten eigenverantwortlich zu sichern und regelmäßig weiterzuentwickeln (BbgSchulG 2002). Hierzu stehen den Lehrkräften und anderen schulischen Akteuren die Angebote der staatlichen Lehrkräftefortbildung zur Verfügung. Zu den Anbietern der staatlichen Lehrkräftefortbildung zählen in Brandenburg das Beratungs- und Unterstützungssystem für Schulen und Schulaufsicht (BUSS), die vier Staatlichen Schulämter, das Landesinstitut für Schule und Medien Berlin-Brandenburg (LISUM) sowie das Ministerium für Bildung, Jugend und Sport (MBJS) (Missal 2019). Die einzelnen Anbieter der staatlichen Lehrkräftefortbildung adressieren mit ihren Angeboten jeweils spezifische Teilgruppen (Lehrkräfte, Schulberater/innen, Schulleitung, Schulaufsicht etc.). Fortbildungen für Lehrkräfte werden primär von den vier Staatlichen Schulämtern organisiert. Die Schulämter berufen hierzu reguläre Lehrkräfte zu Schulberater/ innen, die regionale Fortbildungen planen und durchführen (Missal 2019). Diese Fortbildungsangebote sowie der Angebote der weiteren staatlichen Anbieter werden jeweils in der TIS-Datenbank hinterlegt und bilden die Grundlage für die vorliegende Untersuchung.

\subsection{Datengrundlage}

Für den betreffenden Untersuchungszeitraum wurden aus der TIS-Datenbank insgesamt $n=4910$ Veranstaltungseinträge dokumentiert. Vor der Analyse dieses Daten-

\footnotetext{
2 Im Untersuchungszeitraum (Schuljahr 2016/2017) wurden insgesamt 61 Ersatz- und 364 Ergänzungsangebote unterbreitet. Bei Ersatzangeboten handelt es sich um Veranstaltungen nichtstaatlicher Anbieter für die ein dienstliches Interesse der teilnehmenden Lehrkräfte angenommen werden kann und deren Kosten voll erstattungsfähig sind. Bei Ergänzungsangeboten handelt es sich ebenfalls um Angebote, die nicht von staatlichen Anbietern unterbreitet werden, für die jedoch kein dienstliches Interesse angenommen werden kann und deren Kosten aus diesem Grund auch nicht erstattungsfähig sind.
} 
satzes wurde er in zwei Schritten bereinigt. In einem ersten Schritt wurden Einträge ausgeschlossen, die sich außerhalb des Erkenntnisinteresses dieser Arbeit befinden. Dieses liegt ausschließlich auf schulexternen Lehrkräftefortbildungen. Berücksichtigt werden aus diesem Grund 1) nur Veranstaltungen von staatlichen Anbietern von Lehrkräftefortbildungen (staatliche Schulämter). Ausgeschlossen werden 2) alle schulinternen Veranstaltungen, da diese eine Sonderstellung innehaben. Zum einen gilt für schulinterne Fortbildungen in einigen Fällen eine Präsenzpflicht für Lehrkräfte, zum anderen schließen sie Lehrkräfte anderer Schulen aus, sodass die Zahl der Teilnehmer/innen stark von der entsprechenden Zielgruppe in der Schule und der Schulgröße abhängt. Nicht berücksichtigt wurden zudem 3) alle Weiterbildungs- und Qualifizierungsmaßnahmen, die im Gegensatz zu Fortbildungen das Ziel verfolgen, berufliche Qualifikationen zu vermitteln oder zu erweitern (z. B. die Lehrbefähigung in einem Fach; Richter 2016) sowie Beratungsveranstaltungen und Regionalgruppentagungen, da diese nicht im Sinne der Definition einer Fortbildung der Erweiterung und Aktualisierung von Wissen und Kompetenzen dienen. Betrachtet wurden 4) weiterhin nur Veranstaltungen, die entweder direkt an Lehrkräfte adressiert sind oder diese zumindest nicht ausschließen (z. B. ,,alle Interessierten“); nicht betrachtet werden sollen deshalb Maßnahmen für Fortbildner/innen (z. B. Schulberater/innen) oder Schulleitungen, da es sich hierbei um spezifische Populationen handelt. In einem zweiten Schritt wurde der Datensatz um 5) Fehleinträge sowie ausgefallene Veranstaltungen und 6) Ausreißer bereinigt. Bei Fehleinträgen handelt es sich um doppelte Einträge oder um Veranstaltungen, die neu terminiert wurden. Hinsichtlich der Ausreißer sind wir den Empfehlungen der Trimmed Mean Method (Iglewicz und Hoaglin 1993) gefolgt. Hierzu wurden aus dem Datensatz die Veranstaltungen mit den $5 \%$ meisten Teilnehmer/innen sowie den $5 \%$ längsten Veranstaltungen entfernt, da unplausible Extremwerte den Zusammenhang stark beeinflussen und die Ergebnisse verzerren könnten. Der bereinigte Datensatz umfasste schließlich $n=1330$ schulexterne Lehrerfortbildungen. Bei insgesamt 791 Maßnahmen handelt es sich um Einzelveranstaltungen, 539 Maßnahmen sind dagegen Bestandteil von insgesamt 106 Fortbildungsreihen. Hierbei handelt es sich um mehrere thematisch zusammengehörige Veranstaltungen, welche von einer Person(engruppe) durchgeführt werden, die inhaltlich miteinander verknüpft sind und sich in der Regel über einen längeren Zeitraum erstrecken. Eine gesonderte Analyseeinheit stellen ferner 144 Fortbildungsveranstaltungen dar, die wegen fehlender Nachfrage abgesagt wurden.

\subsection{Beschreibung der interessierenden Variablen}

\subsubsection{Angebotsvariablen von Fortbildungsveranstaltungen}

Entsprechend der Fragestellungen der vorliegenden Untersuchung wurden als Angebotsvariablen zeitliche Merkmale von Fortbildungen analysiert. Insgesamt wurden drei Variablen berücksichtigt, die für jede Fortbildungsveranstaltung in der TIS-Da- 
tenbank hinterlegt sind. ${ }^{3}$ Hierzu zählt die Dauer einer Veranstaltung in Zeitstunden (Forschungsfrage 1). Des Weiteren wurde die Tageszeit der Veranstaltungen in den Blick genommen (Forschungsfrage 2a). Schließlich wurde der Monat, in dem eine Fortbildungsveranstaltung stattfindet, erfasst (Forschungsfrage 2b). Im Rahmen der Datenaufbereitung wurde außerdem eine Variable erzeugt, die anzeigt, ob die Veranstaltung an einem „Schultag“ oder an einem ,unterrichtsfreien Tag“ angeboten wurde (Forschungsfrage 2b). Insgesamt gab es im akademischen Jahr 2016/2017 (01.08.2016 bis 31.07.2017) im Land Brandenburg $n=192$ Schultage und $n=173$ unterrichtsfreie Tage. Als unterrichtsfreie Tage wurden hierbei alle Samstage, Sonntage, alle gesetzlichen Feiertage im Land Brandenburg sowie alle Ferientage definiert.

\subsubsection{Teilnahme an Lehrerfortbildungen}

Zur Beschreibung der Fortbildungsnutzung (Forschungsfrage 1-2b) wurde die Zahl der Teilnehmer/innen verwendet, die in der TIS-Datenbank für jede Veranstaltung hinterlegt ist. Die Teilnehmerzahl ergibt sich dabei wie oben beschrieben aus der Zahl der tatsächlichen Anmeldungen durch in Brandenburg tätige Lehrkräfte.

\subsection{Datenanalyse}

Alle Analysen wurden mit IBM SPSS Statistics 25 durchgeführt. Das analytische Vorgehen war für alle drei Forschungsfragen grundsätzlich identisch: Zunächst wurden zur Beantwortung der jeweils ersten Teilfrage deskriptive Analysen durchgeführt, um die Verteilung des Fortbildungsangebotes zu beschreiben. Hierzu wurden statistische Kennwerte berechnet (Mittelwert, Standardabweichung, Minimum, Maximum). Zur Beantwortung der zweiten Teilfrage nach Zusammenhängen zwischen den zeitlichen Fortbildungsmerkmalen (Dauer, Beginn, Monat) und der Anzahl der Teilnehmer/innen wurden polynomiale Regressionsmodelle genutzt, um lineare und kurvilineare Zusammenhänge abzubilden und gegeneinander zu testen (Kutner et al. 2004). Kurvilineare Zusammenhänge wurden dabei im Besonderen in den Blick genommen, um zu prüfen, ob die Annahme einer Linearität zwischen Merkmalen des Angebots und der Nachfrage Gültigkeit besitzt. Diesbezüglich werden sowohl quadratische als auch kubische Modelle geschätzt, um Funktionen mit einem bzw. zwei Wendepunkten zu identifizieren und somit eine größere Flexibilität der Modellierung zu erreichen. Für diese Analysen diente jeweils ein Veranstaltungsmerkmal (Dauer bzw. Zeitpunkt) als unabhängige Variable und die Teilnehmerzahl der Veranstaltung als abhängige Variable. Zur Validierung der Ergebnisse wurden ferner für alle drei Forschungsfragen einfaktorielle Varianzanalysen durchgeführt, bei denen die Mittelwerte des jeweils interessierenden Merkmals (Dauer, Beginn, Monat) der

\footnotetext{
3 Der Wochentag der Veranstaltung wurde in den Analysen nicht berücksichtigt, da in dem betreffenden Bundesland konkrete Empfehlungen zum Wochentag bestehen. Lehrerfortbildner/innen sind dazu angehalten, Fortbildungsveranstaltungen vorrangig am Dienstag oder Mittwoch stattfinden zu lassen. Aus diesem Grund besteht im Merkmal Wochentag kaum Varianz im Land Brandenburg.
} 
1330 stattgefundenen Veranstaltungen mit denen der 144 wegen fehlender Nachfrage ausgefallenen Veranstaltungen verglichen wurden.

Für die erste Forschungsfrage, die sich auf die Dauer der Veranstaltungen bezog, wurden die Analysen getrennt für Einzelveranstaltungen und Fortbildungsreihen durchgeführt, um differenziertere Aussagen über das Fortbildungsangebot machen zu können. Hierfür wurden die 539 Einzeltermine der 106 Fortbildungsreihen anhand der Veranstaltungsnummer aggregiert. Dabei wurde die Dauer der Einzeltermine, die zu einer Fortbildungsreihe gehören, addiert und das arithmetische Mittel der Teilnehmerzahlen aller Einzeltermine, die zu einer Fortbildungsreihe gehören, gebildet. Zusammenhangsanalysen mit der Dauer werden für Einzelveranstaltungen und Fortbildungsreihen separat durchgeführt, um zu prüfen, ob sich ähnliche Muster für beide Veranstaltungstypen feststellen lassen. Für die zweite und dritte Forschungsfrage, die sich auf den Beginn sowie den Monat der Veranstaltungen beziehen, werden die 1330 Fortbildungsveranstaltungen insgesamt betrachtet, da bei diesen Forschungsfragen der Einzeltermin im Fokus der Untersuchung stand und es inhaltlich nicht plausibel wäre, die Tageszeit oder Monate von verschiedenen Veranstaltungen einer Fortbildungsreihe zu aggregieren.

\section{Ergebnisse}

\subsection{Forschungsfrage 1}

Zur Untersuchung der ersten Forschungsfrage nach der Dauer von Fortbildungsveranstaltungen und etwaigen Zusammenhängen mit der Zahl der Teilnehmer/innen wurden zunächst deskriptive Analysen zur Beschreibung des Angebotes getrennt nach Einzelveranstaltungen und Fortbildungsreihen durchgeführt (Abb. 2). Die Ergebnisse zeigen, dass die Dauer der 791 Einzelveranstaltungen eine durchschnittliche Länge von $M=3,1 \mathrm{~h}(S D=1,5 \mathrm{~h})$ aufweisen. Der Median der Verteilung liegt bei 3,0h. Die 106 Fortbildungsreihen besitzen eine Länge von $M=23,1 \mathrm{~h}(S D=28,5 \mathrm{~h})$. Der Median dieser Verteilung liegt bei $12,0 \mathrm{~h}$. Weiterhin zeigt sich für die wegen fehlender Beteiligung ausgefallen Veranstaltungen eine Länge von $M=2,9 \mathrm{~h}$ $(S D=1,3 \mathrm{~h})$. Die ausgefallenen Veranstaltungen sind dabei bedeutsam kürzer als die Fortbildungsreihen und unterscheiden sich auch deskriptiv in der Länge von Einzelveranstaltungen. Insgesamt zeigen die Ergebnisse, dass Einzelveranstaltungen häufiger angeboten werden und kürzer als Fortbildungsreihen sind. Auch bezüglich der durchschnittlichen Teilnehmerzahl unterscheiden sich Einzelveranstaltungen bedeutsam von Fortbildungsreihen $(F=10,1, d f=1, p<0,01)$. Während Einzelveranstaltungen eine durchschnittliche Teilnehmerzahl von $M=9,8$ Lehrkräften ( $S D=5,4$ Lehrkräfte) aufweisen, nahmen an Fortbildungsreihen im Durchschnitt $M=11,6$ Lehrkräfte $(S D=5,4$ Lehrkräfte) teil.

Um zu untersuchen, ob die Dauer der Fortbildungsangebote prädiktiv für die Zahl der Veranstaltungsteilnehmer/innen ist, wurden sowohl für die Einzelveranstaltungen als auch für die Fortbildungsreihen eine Reihe polynomialer Regressionsmodelle geschätzt (Tab. 1). Die Ergebnisse für die Einzelveranstaltungen weisen auf einen kubischen Zusammenhang der Dauer und der Zahl der Teilnehmer/innen hin. In dem 


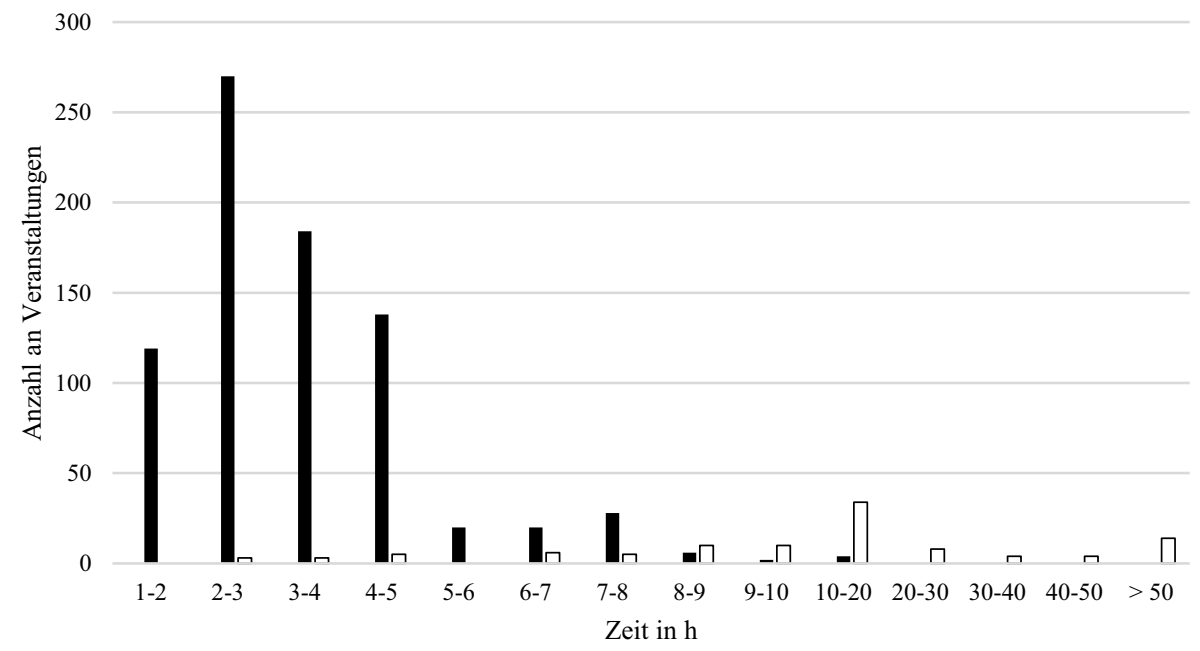

- Einzelveranstaltungen $\square$ Fortbildungsreihe

Abb. 2 Anzahl von Einzelveranstaltungen und Fortbildungsreihen in Abhängigkeit ihrer Dauer

Modell werden sowohl der lineare als auch der quadratische und der kubische Term signifikant $\left(B_{\text {Dauer }}=1,66, p<0,05, B_{\text {Dauer }^{2}}=0,70, p<0,05, B_{\text {Dauer }}{ }^{3}=-0,21, p<0,05\right)$. Die Ergebnisse zeigen jedoch zunächst auch für Fortbildungsreihen einen positiven linearen Effekt der Dauer auf die Zahl der Teilnehmer/innen $\left(B_{\text {Dauer }}=1,62, p<0,05\right)$.

Die statistisch ermittelten Zusammenhänge werden für eine vereinfachte Interpretation in den Abb. 3 und 4 dargestellt. Dort zeigt sich für die Einzelveranstaltungen,

Tab. 1 Ergebnisse der Regressionsmodelle zur Vorhersage der Teilnehmerzahl durch die Veranstaltungsdauer

\begin{tabular}{lllllll}
\hline & Modell 1 & \multicolumn{5}{c}{ Modell 2 } \\
& $B(S E)$ & $\beta$ & $B(S E)$ & $\beta$ & $B(S E)$ & $\beta$ \\
\hline $\begin{array}{lllllll}\text { Einzelveranstaltung } \\
\text { Dauer (h) linear }\end{array}$ & $1,37(0,19)$ & $0,26^{*}$ & $1,76(0,26)$ & $0,33^{*}$ & $1,66(0,26)$ & $0,31^{*}$ \\
$\begin{array}{l}\text { Dauer (h) quadra- } \\
\text { tisch }\end{array}$ & - & - & $-0,22(0,10)$ & $-0,10^{*}$ & $0,70(0,24)$ & $0,33^{*}$ \\
$\begin{array}{l}\text { Dauer (h) kubisch } \\
R^{2}\end{array}$ & - & - & - & - & $-0,21(0,05)$ & $-0,45^{*}$ \\
$\begin{array}{l}\text { Fortbildungsreihen } \\
\text { Dauer (h) linear }\end{array}$ & 0,07 & & 0,07 & & 0,09 & \\
$\begin{array}{l}\text { Dauer (h) quadra- } \\
\text { tisch }\end{array}$ & - & & & & & \\
$\begin{array}{l}\text { Dauer (h) kubisch } \\
R^{2}\end{array}$ & - & - & $-0,08(0,40)$ & $-0,04$ & $-0,91(1,42)$ & $-0,45$ \\
\hline
\end{tabular}

$B$ unstandardisierter Regressionskoeffizient, $S E$ Standardfehler des unstandardisierter Regressionskoeffizienten, $\beta$ standardisierter Regressionskoeffizient, $R^{2}$ Varianzaufklärung des Modells

$* p<0,05$ 


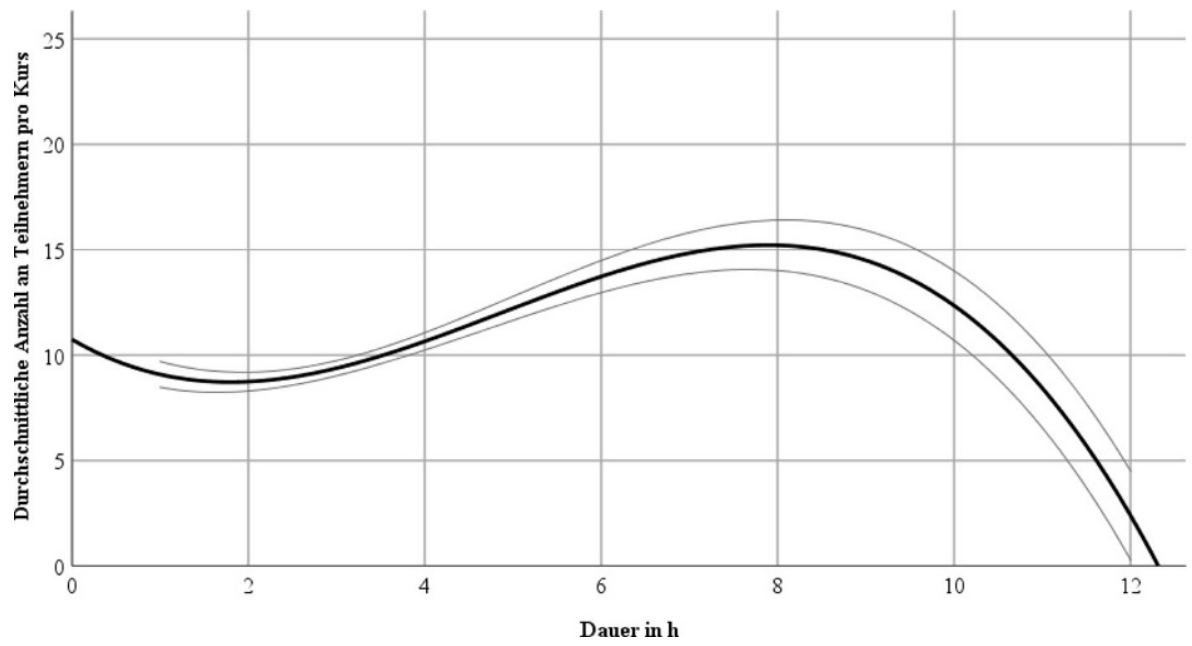

Abb. 3 Teilnehmerzahl an Einzelveranstaltungen als kubische Funktion der Veranstaltungsdauer. (Die schwarze Linie repräsentiert die Funktion, welche durch die Regressionsanalyse vorhergesagt wurde; die graue Linie grenzt das $95 \%$-Konfidenzintervall ab)

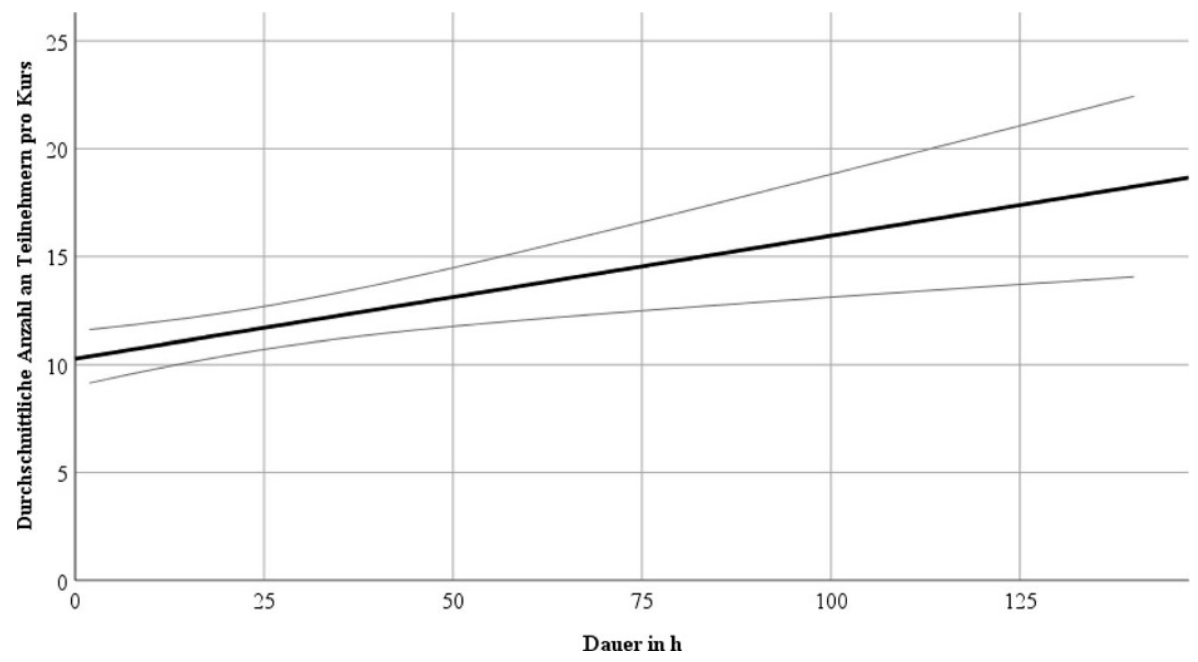

Abb. 4 Teilnehmerzahl an Fortbildungsreihen als lineare Funktion der Veranstaltungsdauer. (Die schwarze Linie repräsentiert die Funktion, welche durch die Regressionsanalyse vorhergesagt wurde; die graue Linie grenzt das $95 \%$-Konfidenzintervall ab)

dass Lehrkräfte eher seltener sehr kurze $(<2 \mathrm{~h})$ oder sehr lange Veranstaltungen aufsuchen ( $>8 \mathrm{~h}$, Abb. 3). In einem Zeitintervall von 2 bis $8 \mathrm{~h}$ nimmt die mittlere Teilnehmerzahl jedoch zu und weist bei $8 \mathrm{~h}$ ihr erwartetes Maximum auf. Dieses Muster deckt sich zu Teilen mit den Ergebnissen für Fortbildungsreihen, für die lediglich ein klarer linearer Zusammenhang beobachtet werden konnte. Das Muster deutet darauf hin, dass Lehrkräfte eher an längeren Fortbildungsreihen partizipie- 
ren (Abb. 4). Ein Maximum an Teilnehmer/innen lässt sich für diese Gruppe an Fortbildungen nicht feststellen.

\subsection{Forschungsfrage $2 a$}

Zur Untersuchung der Forschungsfrage 2a nach dem Beginn von Fortbildungsveranstaltungen und etwaigen Zusammenhängen mit der Zahl der Teilnehmer/innen wurden wiederum zunächst deskriptive Analysen zur Beschreibung der Fortbildungsangebote durchgeführt (Abb. 5). Die Analysen ergaben, dass der Beginn der angebotenen Fortbildungen zwischen 06:00 Uhr und 16:00 Uhr liegt. Im Durchschnitt beginnen die angebotenen Veranstaltungen um $M=11: 42 \mathrm{Uhr}(S D=1,95 \mathrm{~h})$. Der Median der Verteilung liegt bei 12:00 Uhr. Aus Abb. 4 wird ersichtlich, dass lediglich 13,0\% der Angebote vor 10:00 Uhr beginnen und 66,6\% $(n=886)$ der Fortbildungsveranstaltungen in der Zeit zwischen 11:00 und 14:00 Uhr starten. Der Beginn der meisten Veranstaltungen liegt am frühen Nachmittag in der Zeit zwischen 13:00 und 14:00 Uhr $(n=371 ; 27,9 \%)$. Nur 11,7\% $(n=154)$ der angebotenen Fortbildungen begannen nach 14:00 Uhr und damit außerhalb der Unterrichtszeit. Die Befunde der deskriptiven Analysen deuten somit darauf hin, dass Fortbildungsangebote zu großen Teilen eher am späten Vormittag respektive am frühen Nachmittag und eher seltener am Morgen beginnen. Weiterhin zeigen die Analysen für die wegen fehlender Beteiligung ausgefallenen Veranstaltungen einen signifikant späteren durchschnittlichen Beginn um $M=13: 38 \mathrm{Uhr}(S D=1,76 \mathrm{~h})$.

Um zu überprüfen, ob der Beginn einer Fortbildungsveranstaltung prädiktiv für die Teilnehmerzahl ist, wurde erneut eine Reihe polynomialer Regressionsmodelle geschätzt (Tab. 2). Die Ergebnisse weisen auf einen negativen linearen Effekt des Beginns einer Fortbildungsveranstaltung auf die Zahl der Teilnehmer/innen in Mo-

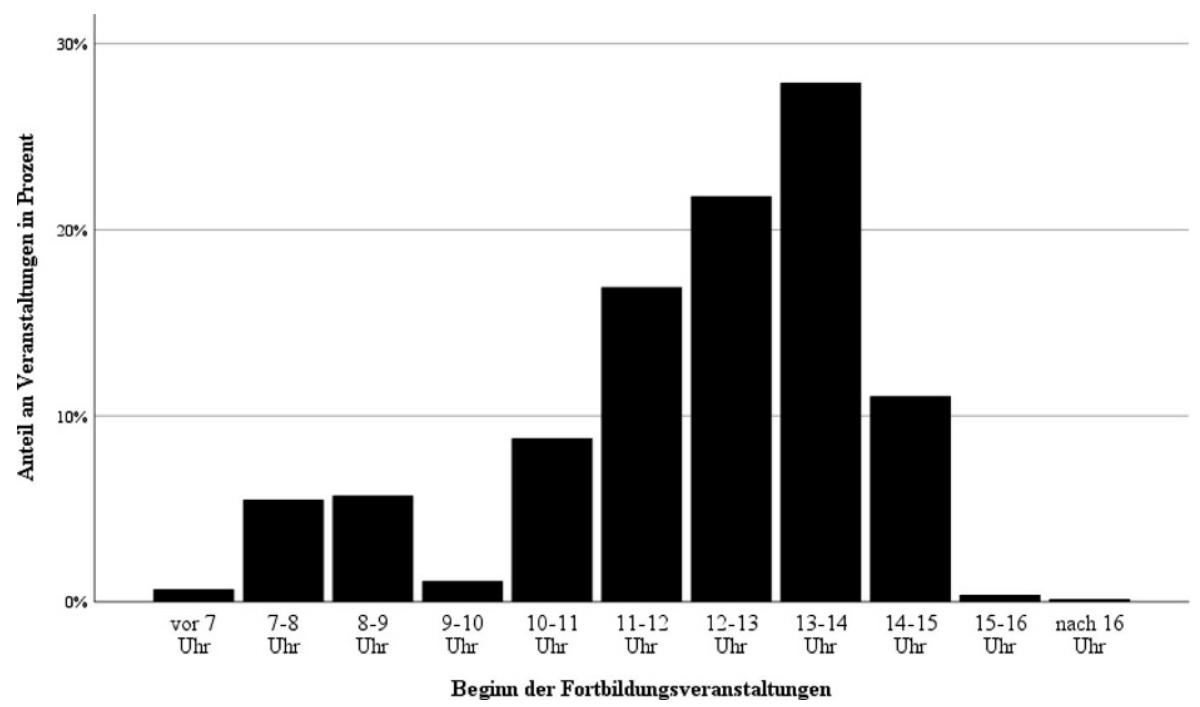

Abb. 5 Anteil von Fortbildungsveranstaltungen in Abhängigkeit des Veranstaltungsbeginns 
Tab. 2 Ergebnisse der Regressionsmodelle zur Vorhersage der Teilnehmerzahl durch den Veranstaltungsbeginn

\begin{tabular}{lllllll}
\hline & Modell 1 & \multicolumn{3}{c}{ Modell 2 } & \multicolumn{3}{c}{ Modell 3 } \\
& $B(S E)$ & $\beta$ & $B(S E)$ & $\beta$ & $B(S E)$ & $\beta$ \\
\hline Beginn linear & $-0,95(0,16)$ & $-0,16^{*}$ & $-0,99(0,21)$ & $-0,17^{*}$ & $-1,28(0,31)$ & $-0,22^{*}$ \\
Beginn quadra- & - & - & $-0,04(0,14)$ & $-0,01$ & $0,19(0,23)$ & 0,05 \\
tisch & - & - & - & & & $0,16(0,13)$ \\
Beginn kubisch & - & - & 0,03 & - & 0,03 & \\
$R^{2}$ & 0,03 & & & & 0,03 \\
\hline
\end{tabular}

$B$ unstandardisierter Regressionskoeffizient, $S E$ Standardfehler des unstandardisierter Regressionskoeffizienten, $\beta$ standardisierter Regressionskoeffizient, $R^{2}$ Varianzaufklärung des Modells $* p<0,05$

dell 1 hin $\left(B_{\text {Beginn }}=-0,95, p<0,05\right)$. Die Prüfung eines weiteren quadratischen und kubischen Effekts zeigt darüber hinaus keine signifikanten Effekte.

Die Befunde verdeutlichen somit, dass der Zusammenhang zwischen dem Beginn eines Fortbildungsangebotes und der Teilnehmerzahl am besten durch eine lineare Funktion vorhergesagt wird (Abb. 6). Demnach partizipieren Lehrkräfte stärker an Fortbildungen, die eher am Tag beginnen. Die erwartete Teilnehmerzahl sinkt von 14 Personen um 6:00 Uhr um ca. 36\% auf 9 Personen um 16:00 Uhr.

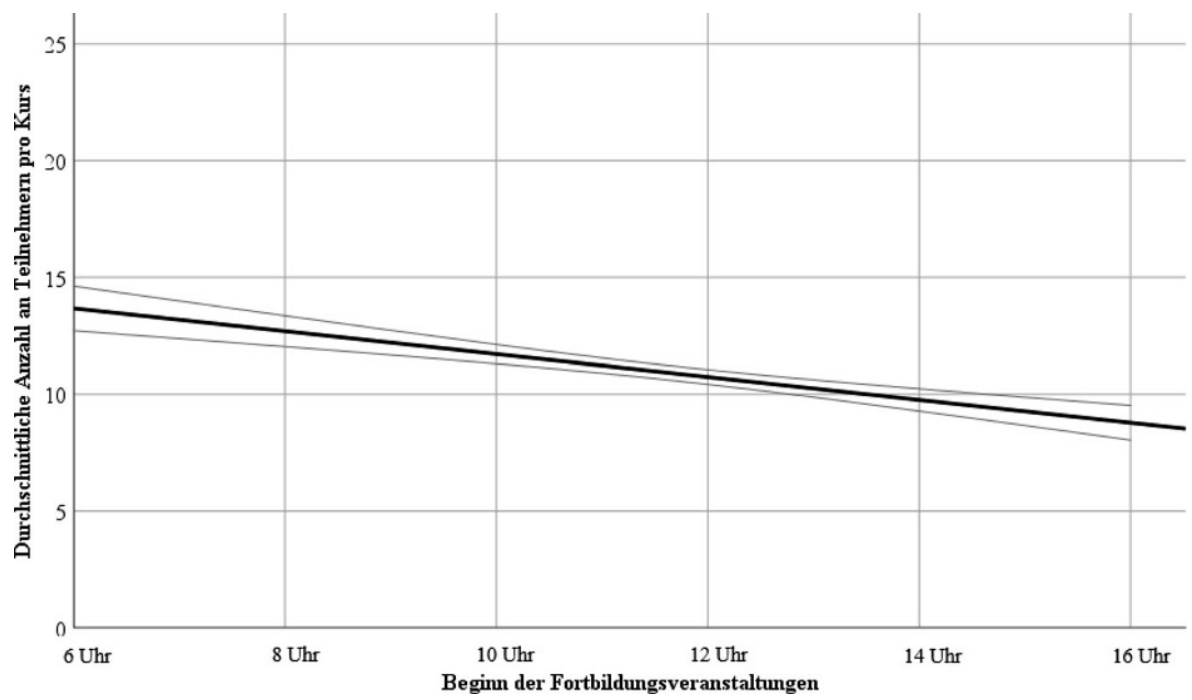

Abb. 6 Teilnehmerzahl an Fortbildungsreihen als lineare Funktion des Veranstaltungsbeginns. (Die schwarze Linie repräsentiert die Funktion, welche durch die Regressionsanalyse vorhergesagt wurde; die graue Linie grenzt das $95 \%$-Konfidenzintervall ab) 


\subsection{Forschungsfrage 2b}

Zur Untersuchung von Forschungsfrage $2 \mathrm{~b}$ nach dem Zeitpunkt der Fortbildungsveranstaltungen innerhalb des Schuljahres und etwaigen Zusammenhängen mit der Zahl der Teilnehmer/innen wurden wie zuvor zunächst deskriptive Analysen zur Beschreibung des Angebots durchgeführt. Dabei wurde überprüft, wie viele Fortbildungsveranstaltungen auf der einen Seite während der Unterrichtszeit und auf der anderen Seite während unterrichtsfreier Tage angeboten wurden. Die Analyse ergab, dass während der 192 Schultage im akademischen Jahr 2016/2017 n=1319 schulexterne Lehrerfortbildungen angeboten wurden. An den 173 unterrichtsfreien Tagen wurden dagegen lediglich $n=11$ schulexterne Lehrerfortbildungen angeboten. Demnach wurden 99,2\% aller Veranstaltungen in der Unterrichtszeit angeboten, welche lediglich etwa die Hälfte aller Tage im Schuljahr ausmacht. Die Analysen zeigen, dass im akademischen Jahr 2016/2017 pro Monat durchschnittlich $M=119,91$ Veranstaltungen ( $S D=60,21$ Veranstaltungen) angeboten wurden (Abb. 7). Die meisten Fortbildungen wurden dabei im November angeboten $(n=244)$ und die wenigsten Fortbildungen wurden im Juli angeboten $(n=31)^{4}$. Die Analysen zeigen zudem, dass Veranstaltungen, die wegen fehlender Beteiligung abgesagt wurden, im Vergleich zu den realisierten Angeboten später im Schuljahr stattgefunden hätten $(F=35,1, d f=1$, $p<0,01)$.

Da die Zahl der Angebote pro Monat durch die Anzahl der unterrichtsfreien Tage pro Monat verzerrt sein könnte, wurde außerdem analysiert, wie viele Fortbildungsveranstaltungen im Durchschnitt pro Schultag und Monat angeboten wurden (Abb. 8). Hierbei wird deutlich, dass die meisten Fortbildungen pro Schultag im November ( $n=11,0$ Angebote pro Schultag) angeboten wurden, die wenigsten Veranstaltungen hingegen im Juni ( $n=2,3$ Angebote pro Schultag).

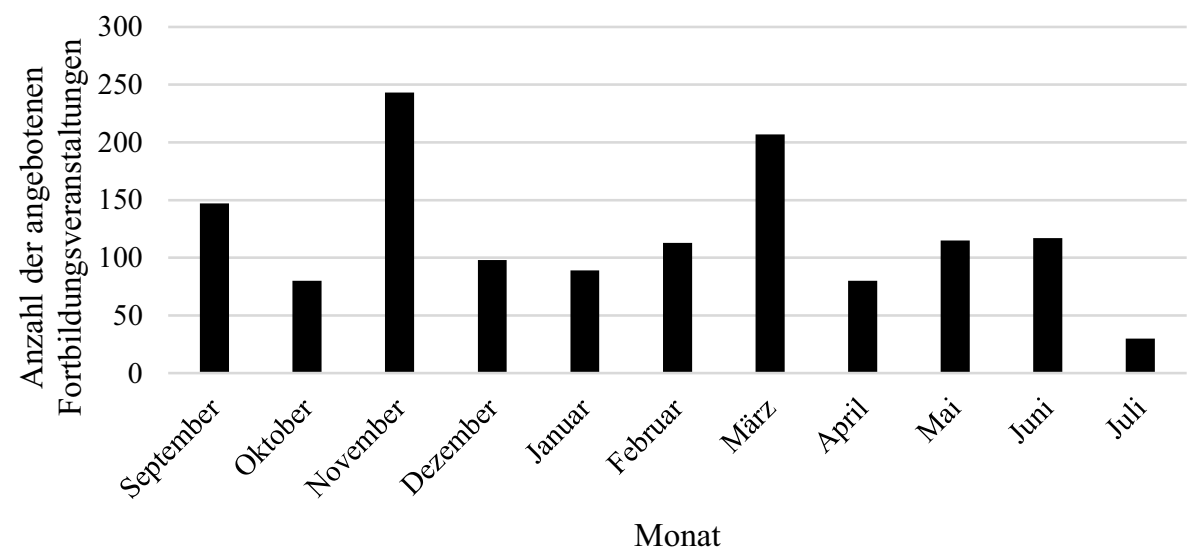

Abb. 7 Anzahl von Fortbildungsveranstaltungen in Abhängigkeit des Monats. (Im Monat August wurde keine Fortbildungsveranstaltung angeboten)

\footnotetext{
4 Im August, dem ersten Monat des akademischen Jahres 2016/2017, wurden keine Fortbildungsveranstaltungen angeboten.
} 


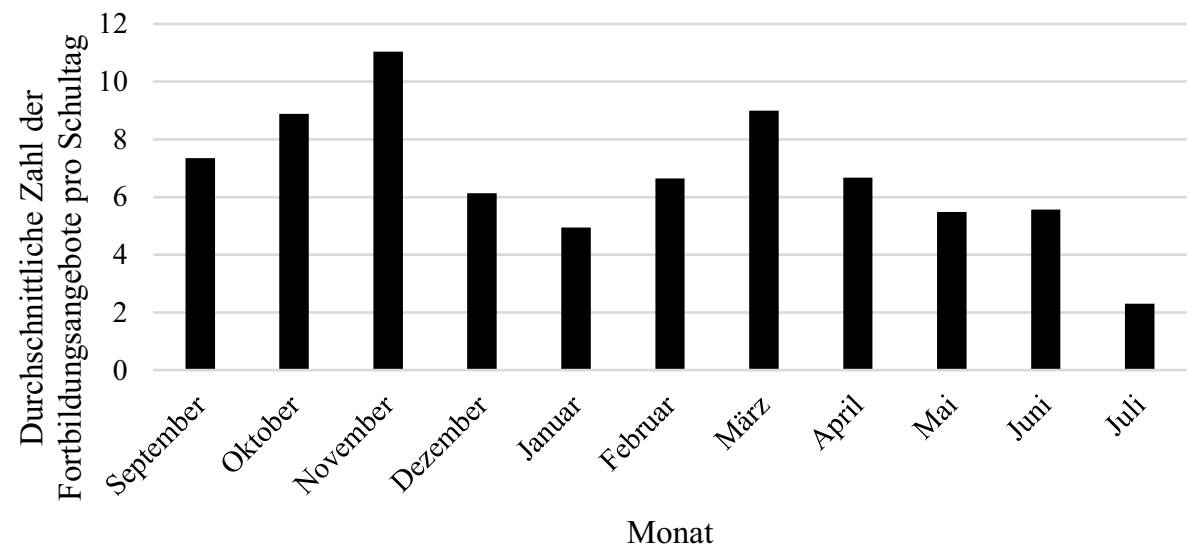

Abb. 8 Durchschnittliche Anzahl von Fortbildungsveranstaltungen pro Schultag in Abhängigkeit des Monats. (Im Monat August wurde keine Fortbildungsveranstaltung angeboten)

Für die Untersuchung der Vorhersagekraft des Monats hinsichtlich der Teilnehmerzahl der Fortbildungsangebote wurden die Monate in einem ersten Schritt rekodiert, sodass dem August als erster Monat des Schuljahres der Wert 1 und dem Juli als letzten Monat des Schuljahres der Wert 12 zugeteilt wurde. Anschließend wurde erneut eine Reihe polynomialer Regressionsmodelle geschätzt. Die Ergebnisse der Analysen sind in Tab. 3 dargestellt. Demnach lässt sich der Zusammenhang zwischen dem Beginn und der Teilnehmerzahl durch einen quadratischen Term am besten beschreiben. In diesem Modell wird sowohl der lineare Term als auch der quadratische Term signifikant $\left(B_{\text {Monat }}=-0,78, p<0,05, B_{\text {Monat }^{2}}=0,41, p<0,05\right)$.

Zur besseren Interpretation des Zusammenhangs wird der Befund in Abb. 9 dargestellt. Demnach nimmt die Teilnehmerzahl an Fortbildungsveranstaltungen im ersten Halbjahr des Schuljahres leicht ab und steigt anschließend wieder leicht an.

Tab. 3 Ergebnisse der Regressionsmodelle zur Vorhersage der Teilnehmerzahl durch den Veranstaltungszeitpunkt innerhalb des Schuljahres

\begin{tabular}{lllllll}
\hline & Modell 1 & & Modell 2 & \multicolumn{3}{c}{ Modell 3 } \\
& $B(S E)$ & $\beta$ & $B(S E)$ & $\beta$ & $B(S E)$ & $\beta$ \\
\hline Monat linear & $-0,74(0,16)$ & $-0,13^{*}$ & $-0,78(0,16)$ & $-0,13^{*}$ & $-0,81(0,37)$ & $-0,14^{*}$ \\
Monat quadra- & - & - & $0,41(0,18)$ & $0,06^{*}$ & $0,40(0,18)$ & $0,06^{*}$ \\
tisch & & - & & & & \\
Monat kubisch & - & - & - & - & $0,02(0,19)$ & 0,01 \\
$R^{2}$ & 0,02 & & 0,02 & & 0,02 & \\
\hline
\end{tabular}

$B$ unstandardisierter Regressionskoeffizient, $S E$ Standardfehler des unstandardisierter Regressionskoeffizienten, $\beta$ standardisierter Regressionskoeffizient, $R^{2}$ Varianzaufklärung des Modells

$* p<0,05$ 


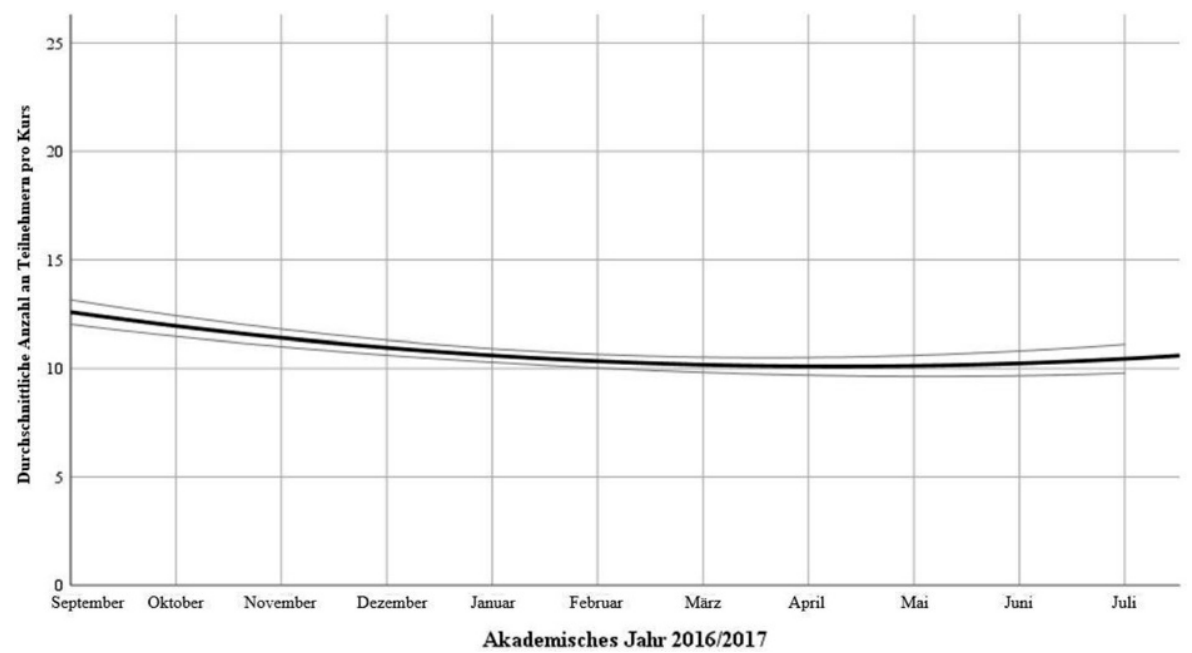

Abb. 9 Teilnehmerzahl an Fortbildungsreihen als quadratische Funktion des Veranstaltungszeitpunktes innerhalb des akademischen Jahres 2016/2017. (Die schwarze Linie repräsentiert die Funktion, welche durch die Regressionsanalyse vorhergesagt wurde; die graue Linie grenzt das $95 \%$-Konfidenzintervall ab)

\section{Diskussion}

Die vorliegende Untersuchung zielte darauf ab, Erkenntnisse über zeitliche Aspekte des Angebots von Lehrerfortbildungen, insbesondere die Dauer und den Zeitpunkt, zu gewinnen und Zusammenhänge zur Nutzung herauszuarbeiten. Für dieses Anliegen wurden die Angebotsdaten der staatlichen Lehrerfortbildung in Brandenburg aus dem akademischen Jahr 2016/2017 ausgewertet. Die wichtigsten Ergebnisse dieser Arbeit lassen sich wie folgt zusammenfassen:

\subsection{Wie lange dauern Fortbildungsveranstaltungen für Lehrkräfte und bestehen Zusammenhänge zwischen der Dauer und der Anzahl der Teilnehmer/innen?}

Es konnte gezeigt werden, dass es sich bei den in Brandenburg angebotenen Lehrerfortbildungen mehrheitlich um Einzelveranstaltungen und seltener um Fortbildungsreihen handelt. Die Deskription aller Angebote zeigte, dass vor allem halbtägige Veranstaltungen dominieren. Diese Befundlage deckt sich mit Ergebnissen aus anderen Bundesländern, wie Bayern (Bayerisches Staatsministerium für Bildung 2017) oder Baden-Württemberg (Cramer et al. 2019) und scheint somit nicht spezifisch für Brandenburg zu sein. Es konnte ferner verdeutlicht werden, dass sowohl die Dauer von Einzelveranstaltungen als auch von Fortbildungsreihen mit der Teilnehmerzahl zusammenhängt. Die Ergebnisse deuten darauf hin, dass Lehrkräfte Einzelveranstaltungen, welche kürzer als 2 oder länger als $8 \mathrm{~h}$ sind, eher seltener aufsuchen. Für Einzelveranstaltungen zwischen 2 und $8 \mathrm{~h}$ zeigte sich jedoch eine Zunahme der Teilnehmerzahl mit steigender Angebotsdauer. Ein ähnlicher Befund ist für Fortbildungsreihen zu konstatieren, für die gezeigt werden konnte, dass Lehrkräfte verstärkt 
an Fortbildungsreihen mit einer höheren zeitlichen Dauer partizipieren. Die Ergebnisse liefern außerdem Hinweise darauf, dass Fortbildungsreihen im Vergleich zu Einzelveranstaltungen stärker nachgefragt werden.

Diese Ergebnisse widersprechen der Arbeitshypothese, die ausgehend von Befunden der allgemeinen Weiterbildungsforschung davon ausging, dass Lehrkräfte zeitlich kürzere Veranstaltungen priorisieren, da diese leichter mit ihrem hohen Arbeitspensum zu verbinden sind (Richter und Pant 2016) und in geringerer Konkurrenz $\mathrm{zu}$ anderen privaten Verpflichtungen stehen dürften (Lobe 2015; Denninger et al. 2017). Sie stehen jedoch in Einklang mit Überlegungen von Kanwischer et al. (2004), wonach Lehrkräfte ganztägige Veranstaltungen bevorzugen, da sie in diesem Falle an diesen Tagen keinen eigenen Unterricht halten müssen und sich stärker auf den eigentlichen Gegenstand der Fortbildung einlassen können. Eine mögliche Erklärung für diesen Befund könnten Erwartungs-Wert-Ansätze bieten (Wigfield und Eccles 2000). Vor diesem Hintergrund kann die Partizipation von Lehrkräften an beruflichen Lerngelegenheiten als leistungsbezogene Aufgabenwahl (z.B. Besuch einer bestimmten Fortbildungsveranstaltung) verstanden werden (Gorges 2015). Die Entscheidung für oder gegen die Teilnahme an beruflichen Lerngelegenheiten hängt dabei maßgeblich mit dem antizipierten Wert zusammen (,Will ich das und warum“, Gorges 2015), den Interessent/innen einer Veranstaltung zuschreiben (Eccles 2005). Dabei werden verschiedene Wertdimensionen unterschieden (z. B. intrinsischer Wert, utilitaristischer Wert, persönlicher Wert), welche die Aufgabenausführung attraktiv machen können (Eccles 1983). Es ist vor diesem Hintergrund plausibel anzunehmen, dass die antizipierte Einschätzung dieser Wertaspekte durch die potentiellen Teilnehmer/innen durchaus auch in Abhängigkeit von Angebotsmerkmalen erfolgt. So ist etwa aus der Forschung zur Fortbildungsqualität bekannt, dass sehr kurze, einmalig stattfindende Veranstaltungen (sog. one-shot Veranstaltungen) nur wenig bis gar nicht wirksam sind (Yoon et al. 2007; Desimone und Garet 2015; DarlingHammond et al. 2017), was beispielsweise ihren utilitaristischen Wert einschränken könnte. Ob Lehrkräfte dies in dieser Art und Weise ebenfalls wahrnehmen, wurde bisher empirisch nicht untersucht. Es ist jedoch nicht abwegig, dass sie sich als Experten/innen für Lehren und Lernen der Bedeutung ausreichend vorhandener Lernzeit durchaus bewusst sind. Diese Annahme könnte die widersprüchlichen Befunde der allgemeinen Weiterbildungsforschung teilweise erklären und wird dadurch untermauert, dass Fortbildungsreihen, die von ihrer konzeptionellen Anlage her eher auf Langfristigkeit ausgelegt sind als Einzelveranstaltungen, eine höhere Teilnehmerzahl aufweisen als Einzelveranstaltungen.

\subsection{Zu welcher Uhrzeit beginnen Fortbildungsveranstaltungen für Lehrkräfte und hängt der Beginn der Veranstaltung mit der Anzahl der Teilnehmer/ innen zusammen?}

Die Ergebnisse der vorliegenden Untersuchung deuten zum einen darauf hin, dass Lehrkräftefortbildungen eher in der Mittagszeit sowie am frühen Nachmittag und damit eher tendenziell außerhalb der regulären Unterrichtszeit angeboten werden. Dieser Befund entspricht den Ergebnissen aus anderen Bundesländern (Amrhein und Badstieber 2015; Bayerisches Staatsministerium für Bildung 2017; DVLfB 2018). 
Zum anderen weisen die Befunde jedoch darauf hin, dass Lehrkräfte eher von Angeboten Gebrauch machen, welche früher am Tag und somit eher in der eigentlichen Unterrichtszeit beginnen. Dies deutet gleichzeitig auf eine grundsätzliche Bereitschaft der Schulleitungen hin, Lehrkräfte trotz unterrichtlicher Verpflichtungen für Fortbildungen freizustellen, wodurch sie ferner ihrer im Schulgesetz verankerten Aufgabe zur Personalentwicklung (Meyer et al. 2019) nachkommen. Die Befunde zeigen weiterhin, dass Lehrkräfte Unterrichtszeit am Vormittag für ihre persönliche Weiterentwicklung nutzen, obwohl der Gesetzgeber Lehrkräfte eher dazu anhält, die Arbeitszeit außerhalb der Unterrichtszeit zu nutzen. Eine mögliche Erklärung für diesen Befund bieten Untersuchungen zur Fortbildungsmotivation von Lehrkräften, die zeigen konnten, dass Lehrkräfte aus verschiedenen Gründen an Fortbildungen partizipieren. Über verschiedene Studien hinweg zeigte sich dabei, dass Lehrkräfte u.a. berichten, Fortbildungen aufzusuchen, um dort in sozialen Kontakt treten zu können oder dem regulären Alltag entfliehen zu wollen (Kao et al. 2011; Rzejak et al. 2014; Richter et al. im Druck). Befunde aus der allgemeinen Weiterbildungsforschung deuten zudem darauf hin, dass Personen. am Nachmittag bedeutsame konkurrierende Verpflichtung (z. B. Kinderbetreuung) wahrnehmen (Denninger et al. 2017). Der Befund deckt sich mit weiteren Befunden der allgemeinen Weiterbildungsforschung, die ebenfalls darauf hindeuten, dass Teilnehmer/innen an beruflicher Bildung bevorzugt Angebote am Vormittag wahrnehmen (Schmidt-Lauff 2008, 2009). Eine mögliche Erklärung für dieses Verhalten bietet die sogenannte Zeitqualität, bei der es sich um die subjektive Zuschreibung eines Wertes für bestimmte Zeiten durch eine Person handelt (Schmidt-Lauff 2010). Studien haben diesbezüglich gezeigt, dass dem Vormittag - anders als dem Nachmittag oder Abend - von Weiterbildungsteilnehmer/innen eine hohe subjektive Aufnahmefähigkeit zugeschrieben wird (Schmidt-Lauff 2008).

\subsection{Zu welchen Zeitpunkten im Jahr werden Fortbildungsveranstaltungen für Lehrkräfte angeboten und hängt dieser Zeitpunkt mit der Anzahl der Teilnehmer/innen zusammen?}

Die statistische Überprüfung dieser Forschungsfrage liefert Hinweise darauf, dass die Zahl der angebotenen Fortbildungsveranstaltungen über das akademische Jahr hinweg ungleich verteilt ist. Besonders eindrücklich zeigte sich, dass insbesondere in der unterrichtsfreien Zeit nur in einem sehr geringen Ausmaß Fortbildungsangebote gemacht werden. Der Befund steht im Einklang mit den Ergebnissen einer Untersuchung des Fortbildungsangebotes in Baden-Württemberg, in der gezeigt wurde, dass nur $1 \%$ des Fortbildungsangebotes während der Schulferien stattfand (Cramer et al. 2019). Die Ergebnisse legen zudem nahe, dass die Bereitschaft, an Fortbildungen zu partizipieren, im Laufe des Schuljahres schwankt. Bei genauer Betrachtung zeigt sich zudem, dass sich die Beteiligung der Lehrkräfte an Fortbildungen mithilfe einer U-Form beschreiben lässt. Demnach partizipieren Lehrkräfte am Anfang und am Ende des Schuljahres stärker an Fortbildungen als zur Schuljahresmitte. Dieses Muster deckt sich nur zu Teilen mit der eingangs formulierten Hypothese, wonach die Zahl der Teilnehmer/innen unmittelbar vor den Zeugnissen (Januar, Juni) wegen der als hoch angenommen Arbeitsbelastung besonders gering sind (Mußmann et al. 
2016). Letztlich konnte im Rahmen dieser Arbeit nicht für die realen Arbeitszeiten der brandenburgischen Lehrkräfte im Schuljahr 2016/2017 kontrolliert werden und es ist zumindest plausibel anzunehmen, dass es neben den arbeitsreichen Phasen jeweils vor den Zeugnissen noch weitere arbeitsintensive Phasen gibt. Überhaupt deuten Befunde zur Arbeitszeit von Lehrkräften auf einen insgesamt hohen zeitlichen Umfang hin (Richter und Pant 2016).

Die vorliegenden Befunde erweitern den bisherigen Forschungsstand zur dritten Phase der Lehrkräftebildung substantiell, da es sich bei dieser Arbeit um eine der ersten handelt, die auf Grundlage einer vollständigen Übersicht über alle staatlichen Angebote eines Bundeslandes die zeitlichen Merkmale von Fortbildungen systematisch analysierte. Es konnte gezeigt werden, dass es sich bei einem Großteil des Fortbildungsangebotes vor allem um halbtägige Veranstaltungen handelt, die eher in der Mittagszeit bzw. am frühen Nachmittag und damit eher nicht in der schulischen Kernarbeitszeit beginnen. Höhere Teilnehmerzahlen konnten dagegen vor allem für zeitlich längere Veranstaltungen gefunden werden, die in der Regel bereits am Vormittag während der eigentlichen Unterrichtszeit beginnen. An dieser Stelle ist kritisch anzumerken, dass zwischen dem Beginn und der Dauer einer Veranstaltung aus organisatorischen und zeitlogischen Gründen ein Zusammenhang besteht. Dessen ungeachtet weisen die Ergebnisse auch darauf hin, dass sich die Zahl der Fortbildungsangebote sowie die Beteiligung der Lehrkräfte an Fortbildungen innerhalb eines Schuljahres systematisch voneinander unterscheidet. Ob Lehrkräfte weniger arbeitsreiche Phasen, wie zum Beispiel Schulferien, dazu nutzen würden, um sich fortzubilden oder ob das geringe Fortbildungsangebot als Resultat einer geringen Nachfrage in der Vergangenheit zu verstehen ist, kann dagegen auf Grundlage der vorliegenden Untersuchung nicht beantwortet werden, da mit wenigen Ausnahmen keine Fortbildungsangebote in den Ferien zu verzeichnen waren.

\subsection{Limitationen}

Die vorgestellten Befunde sind nicht generalisierbar für das Fortbildungsangebot und die Fortbildungsnutzung in Deutschland, weshalb an dieser Stelle Einschränkungen bezüglich des Aussagegehaltes der Untersuchung gemacht werden. So fällt bei der Betrachtung der insgesamt drei Regressionsmodelle auf, dass die Zahl der Teilnehmer/innen nur zu einem geringen Ausmaß durch die Merkmale Dauer und Zeitpunkt am Tag und innerhalb des Schuljahres vorhergesagt werden kann. Berücksichtigt man an dieser Stelle jedoch den Forschungsstand zur Fortbildungsnutzung wird deutlich, dass es sich bei der Entscheidung einer Lehrkraft für oder gegen die Teilnahme an einer Fortbildungsveranstaltung um einen hoch komplexen Aushandlungsprozess handelt, welcher multifaktoriell auch durch Merkmale der Person und des Kontextes beeinflusst wird (vgl. Gorges 2015). Die Erklärung der realisierten Nachfrage kann somit nicht als reiner Ausdruck individueller Präferenzen und Entscheidungen (Selbstselektion) verstanden werden, sondern unterliegt nach Boeren et al. (2010) einem komplexen Interaktionsprozess verschiedener Akteure auf der Mikro- (z. B. Individuum), Meso- (z. B. Landesinstitut) und Makroebene (z.B. rechtliche Genehmigung durch die Schulleitung) und somit einer gewissen Fremdselektion. Einschränkend sei weiterhin erwähnt, dass der zugrundeliegende Datensatz 
keine Individualdaten für die einzelnen Teilnehmer/innen enthält. Es können somit keine Aussagen zu individuellen Fortbildungsaktivitäten, wie beispielsweise der mehrmaligen Teilnahme an verschiedenen Fortbildungen, gemacht werden, die gegebenenfalls Einfluss auf die Ergebnisse haben könnten. Eine weitere Einschränkung der vorliegenden Untersuchung besteht darin, dass nur das Fortbildungsangebot eines Bundeslandes und eines Schuljahres untersucht wurde. Inwiefern sich die hier gefundenen Muster auch auf andere Bundesländer übertragen lassen, kann an dieser Stelle nicht beantwortet werden. Schließlich ist außerdem darauf hinzuweisen, dass die vorliegende Untersuchung ausschließlich schulexterne Angebote, die sich explizit an Lehrerinnen und Lehrer gerichtet haben, in den Blick genommen hat. Weitere Untersuchungen müssten vor diesem Hintergrund prüfen, inwiefern sich die Befunde auch auf schulinterne Angebote sowie Veranstaltungen für andere schulische Akteur/innen (z. B. Schulleitungen) übertragen lassen.

\subsection{Implikationen}

Dieser Einschränkungen ungeachtet konnte die vorliegende Studie auf Grundlage einer bisher wenig genutzten Datenquelle neue Befunde aufzeigen, welche Implikationen sowohl für weitere Studien zum Verhältnis von Fortbildungsangebot und -nutzung als auch für die Fortbildungsplanung und Bedarfsermittlung liefern. So konnte gezeigt werden, dass mithilfe vorhandener Angebotsdaten einerseits auf Merkmale von Fortbildungsangeboten geschlossen werden kann und Zusammenhänge zwischen den Angebotsmerkmalen und der Fortbildungsbeteiligung untersucht werden können. Mit Blick auf die vorhandene Datenlage wäre es denkbar, neben zeitlichen Merkmalen auch andere organisatorische Angebotsmerkmale, wie den Veranstaltungsort zu adressieren, um zu prüfen, ob gegebenenfalls Interaktionen mit zeitlichen Veranstaltungsmerkmalen bestehen und Zusammenhänge mit der Teilnehmerzahl zu beobachten sind. Weiterhin könnten auch inhaltliche (z. B. curricularer Bezug) und Prozessmerkmale (z. B. aktives Lernen; vgl. Richter 2016) von Veranstaltungen, und deren Vorhersagekraft für die Fortbildungsnutzung in den Blick zu nehmen. Durch derartige Untersuchungen ließe sich die in verschiedenen Modellen postulierte Interdependenz von Angebot und Nachfrage (Boeren et al. 2010) empirisch überprüfen. Die auf diese Weise gewonnen Erkenntnisse könnten dabei helfen, die Interaktion von Angebot und Nachfrage gegebenenfalls zu optimieren und die Reichweite von Fortbildungen zu erhöhen. Diesbezüglich sei jedoch kritisch angemerkt, dass eine reine Erweiterung des Angebotes aus theoretischer Sicht nicht zwingend mit einer höheren Fortbildungsnutzung seitens der Lehrkräfte einhergehen muss (Boeren et al. 2010).

Um den Interaktionsprozess von Nachfrage und Angebot zu unterstützen, können auf Grundlage der vorliegenden Befunde mögliche Implikationen abgeleitet werden, deren Wirksamkeit empirisch zu überprüfen ist: 1) Das weitestgehende Fehlen von Fortbildungsangeboten in der unterrichtsfreien Zeit, insbesondere während der Schulferien, sollte überwunden werden. Lehrkräfte sollten die Möglichkeit haben, auch in weniger arbeitsreichen Phasen, wie etwa den Ferien, an Fortbildungen teilnehmen zu können (Daus et al. 2004). 2) Bei der Mehrzahl der angebotenen Fortbildungsveranstaltungen handelt es sich um kurze one-shot Veranstaltungen. Diese 
werden in der einschlägigen Forschungsliteratur kritisch betrachtet, da sie nur wenig Lernzeit zur Verfügung stellen, welche für nachhaltiges Lernen notwendig ist (Yoon et al. 2007; Desimone und Garet 2015; Darling-Hammond et al. 2017). Die Befunde der vorliegenden Untersuchung zeigen zudem, dass Veranstaltungen mit größerem zeitlichen Umfang höhere Teilnehmerzahlen aufweisen. Vor diesem Hintergrund könnte geprüft werden, den Anteil längerfristig angelegter Fortbildungsveranstaltungen zu erhöhen.

Abschließend lässt sich festhalten, dass die vorliegende Studie zeitliche Merkmale von Fortbildungsveranstaltungen als eine bedeutsame Determinante der Fortbildungsnutzung durch Lehrkräfte identifizieren konnte. Um zukünftig wirksame Schlussfolgerungen für die Angebotsplanung ableiten zu können, wäre es wünschenswert, weitere Angebotsmerkmale, wie den Fortbildungsinhalt oder den Veranstaltungsort, in den Blick zu nehmen, um die Passung von Angebot und Nachfrage nachhaltig zu steigern.

Funding Open Access funding provided by Projekt DEAL.

Open Access Dieser Artikel wird unter der Creative Commons Namensnennung 4.0 International Lizenz veröffentlicht, welche die Nutzung, Vervielfältigung, Bearbeitung, Verbreitung und Wiedergabe in jeglichem Medium und Format erlaubt, sofern Sie den/die ursprünglichen Autor(en) und die Quelle ordnungsgemäß nennen, einen Link zur Creative Commons Lizenz beifügen und angeben, ob Änderungen vorgenommen wurden.

Die in diesem Artikel enthaltenen Bilder und sonstiges Drittmaterial unterliegen ebenfalls der genannten Creative Commons Lizenz, sofern sich aus der Abbildungslegende nichts anderes ergibt. Sofern das betreffende Material nicht unter der genannten Creative Commons Lizenz steht und die betreffende Handlung nicht nach gesetzlichen Vorschriften erlaubt ist, ist für die oben aufgeführten Weiterverwendungen des Materials die Einwilligung des jeweiligen Rechteinhabers einzuholen.

Weitere Details zur Lizenz entnehmen Sie bitte der Lizenzinformation auf http://creativecommons.org/ licenses/by/4.0/deed.de.

\section{Literatur}

Altrichter, H. (2010). Lehrerfortbildung im Kontext von Veränderungen im Schulwesen. In F. H. Müller, A. Eichenberger, M. Lüders \& J. Mayr (Hrsg.), Lehrerinnen und Lehrer lernen. Konzepte und Befunde zur Lehrerfortbildung (S. 17-34). Münster: Waxmann.

Amrhein, B., \& Badstieber, B. (2015). Lehrerfortbildungen zur Inklusion - eine Trendanalyse. https:// www.bertelsmann-stiftung.de/de/publikationen/publikation/did/lehrerfortbildungen-zu-inklusioneine-trendanalyse/. Zugegriffen: 18. Juli 2018.

Baert, H., de Rick, K., \& van Valckenborgh, K. (2006). Towards the conceptualization of "Learning climate". In R. V. de Castro, P. Guimarães \& A. V. Sancho (Hrsg.), Adult education. New routes in a new landscape (S. 87-111). Braga: University of Minho.

Bayerisches Staatsministerium für Bildung (2017). Schule und Bildung in Bayern 2017. Zahlen und Fakten.

BbgSchulG (2002). Gesetz über die Schulen im Land Brandenburg (Brandenburgisches Schulgesetz BbgSchulG) In der Fassung der Bekanntmachung vom 2. August 2002. Zuletzt geändert durch Artikel 4 des Gesetzes vom 18. Dezember 2018.

Beck, C., \& Ullrich, H. (1996). Fortbildungsinteressen von Lehrenden. Ergebnisse einer repräsentativen Befragung. Die Deutsche Schule, 88(2), 198-213.

Boeren, E., Nicaise, I., \& Baert, H. (2010). Theoretical models of participation in adult education: the need for an integrated model. International Journal of Lifelong Education, 29(1), 45-61.

Bruno, R., Ashby, S., \& Manzo, F. (2012). Beyond the classroom: an analysis of a chicago public school teacher's actual workday. https://www.ctunet.com/blog/text/Beyond-the-Classrooom-Study-April2012-University-of-Illinois-Labor-and-Employment-Relations-for-merge.pdf. Zugegriffen: 18. Juli 2018. 
Collinson, V., \& Fedoruk Cook, T. (2001). "I don't have enough time"-teachers' interpretations of time as a key to learning and school change. Journal of Educational Administration, 39(3), 266-281.

Cramer, C., Johannmeyer, K., \& Drahmann, M. (2019). Fortbildungen von Lehrerinnen und Lehrern in Baden-Württemberg. Tübingen: GO Druck Media.

Darling-Hammond, L., Hyler, M.E., \& Gardner, M. (2017). Effective teacher professional development. https://learningpolicyinstitute.org/sites/default/files/product-files/Effective_Teacher_Professional_ Development_REPORT.pdf. Zugegriffen: 18. Juli 2018.

Daus, J., Pietzner, V., Höner, K., Scheuer, R., Melle, I., Neu, C., Schmidt, S., \& Bader, H. J. (2004). Untersuchung des Fortbildungsverhaltens und der Fortbildungswünsche von Chemielehrerinnen und Chemielehrern. CHEMKON, 11(2), 79-85.

Denninger, A., Kahl, R., \& Präßler, S. (2017). Individuumsbezogene Zeitbudgetstudie - Konzeptionen zur Erhebung der Zeitverausgabung von Teilnehmenden wissenschaftlicher Weiterbildung. In W. Seitter (Hrsg.), Zeit in der wissenschaftlichen Weiterbildung (S. 59-93). Wiesbaden: Springer.

Desimone, L. M., \& Garet, M. S. (2015). Best practices in teachers' professional development in the United States. Psychology, Society, \& Education, 7(3), 252.

DVLfB (2018). Recherchen für eine Bestandsaufnahme der Lehrkräftefortbildung in Deutschland. Ergebnisse des Projektes Qualitätsentwicklung in der Lehrkräftefortbildung (Teil 1, forum Lehrerfortbildung, Bd. 47). Berlin: Deutscher Verein zur Förderung der Lehre-rinnen und Lehrerfortbildung e.V. (DVLfB).

Eccles, J.S. (1983). Expectancies, values and academic behaviors. In J. T. Spence (Hrsg.), Achievement and achievement motives (S. 75-146). San Francisco: Freeman.

Eccles, J.S. (2005). Subjective task value and the Eccles et al. model of achievement-related choices. In A. J. Elliot \& C. S. Dweck (Hrsg.), Handbook of competence and motivation (S. 105-121). New York: Guilford.

Gerlach, F. M., \& Beyer, M. (1999). Ärztliche Fortbildung aus der Sicht niedergelassener Ärztinnen und Ärzte-repräsentative Ergebnisse aus Bremen und Sachsen-Anhalt. Zeitschrift für Ärztliche Fortbildung und Qualitätssicherung, 93, 581-590.

Gorges, J. (2015). Warum (nicht) an Weiterbildung teilnehmen? In J. Gorges, A. Gegenfurtner \& H. Kuper (Hrsg.), Motivationsforschung im Weiterbildungskontext (Zeitschrift für Erziehungswissenschaft: Sonderheft 30, S. 9-28). Wiesbaden: Springer.

Gorozidis, G., \& Papaioannou, A. G. (2014). Teachers' motivation to participate in training and to implement innovations. Teaching and Teacher Education, 39, 1-11.

Graudenz, I., Plath, I., \& Kodron, C. (1995). Lehrerfortbildung auf dem Prüfstand: Erfahrungen, Wirkungen, Erwartungen. Baden-Baden: Nomos.

Heintze, C., Matysiak-Klose, D., \& Braun, V. (2005). Wahrnehmung von Fortbildungsangeboten aus Sicht von Hausärzten: Eine qualitative Befragung von Allgemeinärzten und hausärztlich tätigen Internisten aus Berlin. Zeitschrift für Ärztliche Fortbildung und Qualität im Gesundheitswesen, 99(7), 437-442.

Hoffmann, L., \& Richter, D. (2016). Aspekte der Aus- und Fortbildung von Deutsch- und Englischlehrkräften im Ländervergleich. In P. Stanat, K. Böhme, S. Schipolowski \& N. Haag (Hrsg.), IQB-Bildungstrend 2015. Sprachliche Kompetenzen am Ende der 9. Jahrgangsstufe im zweiten Ländervergleich (S. 481-501). Münster: Waxmann.

Iglewicz, B., \& Hoaglin, D. C. (1993). ASQC basic references in quality control. v. 16: how to detect and handle outliers. Milwaukee: ASQC Quality.

Jäger, R. S., \& Bodensohn, R. (2007). Bericht zur Befragung von Mathematiklehrkräften: Die Situation der Lehrerfortbildung im Fach Mathematik aus der Sicht der Lehrkräfte. https://dzlm.de/files/uploads/ 17_01_07_mathematiklehrerbefragung.pdf. Zugegriffen: 18. Juli 2018.

Kanwischer, D., Köhler, P., \& Gödde, U. (2004). Der Lehrer ist das Curriculum!? Eine Studie zu Fortbildungsverhalten, Fachverständnis und Lehrstilen Thüringer Geographielehrer. Bad Berka: ThILLM.

Kao, C.-P., Wu, Y.-T., \& Tsai, C.-C. (2011). Elementary school teachers' motivation toward web-based professional development, and the relationship with Internet self-efficacy and belief about web-based learning. Teaching and Teacher Education, 27(2), 406-415.

KMK (2000in). 291. Plenarsitzung der Kultusministerkonferenz am 05.Oktober 2000 in Bremen. https:// www.kmk.org/presse/pressearchiv/mitteilung/291-plenarsitzung-der-kultusministerkonferenz-am05oktober-2000-in-bremen.html. Zugegriffen: 18. Juli 2018.

Kutner, M.H., Nachtsheim, C., Neter, J., \& Li, W. (2004). Applied linear statistical models. New York: McGraw-Hill.

Lipowsky, F. (2014). Theoretische Perspektiven und empirische Befunde zur Wirksamkeit von Lehrerfortund -weiterbildung. In E. Terhart, H. Bennewitz \& M. Rothland (Hrsg.), Handbuch der Forschung zum Lehrerberuf (S. 398-417). Münster: Waxmann. 
Lobe, C. (2015). Hochschulweiterbildung als biografische Transition. Teilnehmerperspektiven auf berufsbegleitende Studienangebote (Lernweltforschung, Bd. 20). Wiesbaden: Springer VS. zugl. Bielefeld, Univ., Diss.

Loucks-Horsley, S., Stiles, K., \& Hewson, P. (1996). Principles of effective professional development for mathematics and science education: a synthesis of standards. NISE Brief, 1(1), 1-8.

Meyer, A., Richter, D., Marx, A., \& Hartung-Beck, V. (2019). Welche Aufgaben haben Schulleitungen heute? Eine Analyse von Schulleitungsaufgaben im innerdeutschen Vergleich. Zeitschrift für Bildungsverwaltung, 35(2), 23-44.

Missal, S. (2019). Erfolgreiche Konzepte der Weiterbildung von Lehrkräften. Zur Fortbildung von Lehrkräften und Führungskräften im schulischen Kontext in Brandenburg. In N. McElvany, F. Schwabe, W. Bos \& H. G. Holtappels (Hrsg.), Lehrerbildung - Potentiale und Herausforderungen in den drei Phasen (1. Aufl. IFS-Bildungsdialoge, Bd. 3, S. 109-126). Münster: Waxmann.

Mußmann, F., Riethmüller, M., \& Hardwig, T. (2016). Niedersächsische Arbeitszeitstudie Lehrkräfte an öffentlichen Schulen 2015/2016. http://webdoc.sub.gwdg.de/pub/mon/2016/5-mussmann.pdf. Zugegriffen: 21. Jan. 2020.

Nahrstedt, W., \& Brinkmann, D. (1998). Neue Zeitfenster für Weiterbildung. Temporale Muster der Angebotsgestaltung und Zeitpräferenzen der Teilnehmer im Wandel. Abschlußbericht des Forschungsprojektes: Entwicklung und begleitende Untersuchung von neuen Konzepten der Erwachsenenbildung unter besonderer Berücksichtigung des Aspekts des lebenslangen Lernens und des institutionellen Umgangs mit veränderten temporalen Mustern der Angebotsnutzung. Bielefeld: IFKA.

Nolda, S. (2009). Programmanalyse - Methoden und Forschungen. In R. Tippelt \& A. von Hippel (Hrsg.), Handbuch Erwachsenenbildung/Weiterbildung (S. 293-307). Wiesbaden: VS.

Philipp, A., \& Kunter, M. (2013). How do teachers spend their time? A study on teachers' strategies of selection, optimisation, and compensation over their career cycle. Teaching and Teacher Education, $35,1-12$.

Präßler, S. (2015). Bedarfsanalyse. Forschungsbericht zu Bedarfen individueller Zielgruppen. In W. Seitter, M. Schemmann \& U. Vossebein (Hrsg.), Zielgruppen in der wissenschaftlichen Weiterbildung: Empirische Studien zu Bedarf, Potential und Akzeptanz (S. 61-187). Wiesbaden: VS.

Richter, D. (2016). Lehrerinnen und Lehrer lernen: Fort- und Weiterbildung im Lehrerberuf. In M. Rothland (Hrsg.), Beruf Lehrer/Lehrerin. Ein Studienbuch (S. 245-260). Stuttgart: UTB.

Richter, D., \& Pant, H. A. (2016). Lehrerkooperation in Deutschland. Eine Studie zu kooperativen Arbeitsbeziehungen bei Lehrkräften der Sekundarstufe I. Gütersloh: Bertelsmann Stiftung.

Richter, D., Kleinknecht, M., \& Gröschner, A. (2019). What motivates teachers to participate in professional development? An empirical investigation of motivational orientations and the uptake of formal learning opportunities. Teaching and Teacher Education, 86, 102929. https://doi.org/10.1016/j.tate. 2019.102929.

Richter, D., Kuhl, P., Haag, N., \& Pant, H. A. (2013). Aspekte der Aus- und Fortbildung von Mathematik- und Naturwissenschaftslehrkräften im Ländervergleich. In H. A. Pant, P. Stanat, U. Schroeders, A. Roppelt, T. Siegle \& C. Pohlmann (Hrsg.), IQB-Ländervergleich 2012. Mathematische und naturwissenschaftliche Kompetenzen am Ende der Sekundarstufe I (S. 367-390). Münster: Waxmann.

Richter, D., Kuhl, P., Reimers, H., \& Pant, H. A. (2012). Aspekte der Aus- und Fortbildung von Lehrkräften in der Primarstufe. In P. Stanat (Hrsg.), Kompetenzen von Schülerinnen und Schülern am Ende der vierten Jahrgangsstufe in den Fächern Deutsch und Mathematik. Ergebnisse des IQB-Ländervergleichs 2011 (S. 237-250). Münster: Waxmann.

Richter, D., Kunter, M., Anders, Y., Klusmann, U., Lüdtke, O., \& Baumert, J. (2010). Inhalte und Prädiktoren beruflicher Fortbildung von Mathematiklehrkräften. Empirische Pädagogik, 24(2), 151-168.

Richter, D., Kunter, M., Klusmann, U., Lüdtke, O., \& Baumert, J. (2011). Professional development across the teaching career. Teachers' uptake of formal and informal learning opportunities. Teaching and Teacher Education, 27(1), 116-126.

Richter, E., Richter, D., \& Marx, A. (2018). Was hindert Lehrkräfte an Fortbildungen teilzunehmen? Zeitschrift für Erziehungswissenschaft, 21(5), 1021-1043. https://doi.org/10.1007/s11618-018-0820-4.

Rzejak, D., Künsting, J., Lipowsky, F., Fischer, E., Dezhgahi, U., \& Reichhardt, A. (2014). Facetten der Lehrerfortbildungsmotivation. Eine faktorenanalytische Betrachtung. Jero, 6(1), 139-159.

Schaper, N. (2019). Aus- und Weiterbildung: Konzepte der Trainigsforschung. In F.W. Nerdinger, G. Blickle \& N. Schaper (Hrsg.), Springer-Lehrbuch: Arbeits- und Organisationspsychologie (4. Aufl,. S. 509-539). Heidelberg: Springer.

Scheffer, D., \& Kuhl, J. (2010). Volitionale Prozesse und Zielverfolgung. In U. Kleinbeck, K.-H. Schmidt \& N.-P. Birbaumer (Hrsg.), Arbeitspsychologie. Enzyklopädie der Psychologie Praxisgebiete Wirtschafts-, Organisations- und Arbeitspsychologie (Bd. 1, S. 89-137). Göttingen: Hogrefe. 
Schmidt-Lauff, S. (2008). Zeit für Bildung im Erwachsenenalter. Interdisziplinäre und empirische Zugänge. Münster: Waxmann.

Schmidt-Lauff, S. (2009). Zeitfragen und Temporalität in der Erwachsenenbildung. In R. Tippelt \& A. von Hippel (Hrsg.), Handbuch Erwachsenenbildung/Weiterbildung (S. 213-228). Wiesbaden: VS.

Schmidt-Lauff, S. (2010). Ökonomisierung von Lernzeit. Zeit in der betrieblichen Weiterbildung. Zeitschrift für Pädagogik, 56(3), 355-365.

Statistisches Bundesamt (2015). Zeitverwendungserhebung. Aktivitäten in Stunden und Minuten für ausgewählte Personengruppen. https://www.destatis.de/DE/Publikationen/Thematisch/ EinkommenKonsumLebensbedingungen/Zeitbudgeterhebung/Zeitverwendung5639102139004.pdf; jsessionid=4B8A541A71 A52C0C3CD0F827FC395EE9.InternetLive1?_blob=publicationFile. Zugegriffen: 18. Juli 2018.

US Department of Education (1995). Building bridges: the mission and principles of professional development.

Wei, R.C., Darling-Hammond, L., \& Adamson, F. (2010). Professional development in the United States: trends and challanges. Dallas: National Staff Development Council.

Wigfield, A., \& Eccles, J.S. (2000). Expectancy-value theory of achievement motivation. Contemporary educational psychology, 25(1), 68-81.

Wolf, W., Göbel-Lehnert, U., \& Chroust, P. (1997). Lehrerfortbildung in Hessen. Marburg: Hessisches Institut für Lehrerbildung.

Wolf, W., Göbel-Lehnert, U., \& Chroust, P. (1999). Fortbildung der Lehrerinnen und Lehrer. Eine Bilanz ihrer Formen und Wirkungen anhand empirischer Untersuchungen. Die Deutsche Schule, 91(4), $451-467$.

Yoon, K. S., Duncan, T., Lee, S. W.-Y., Scarloss, B., \& Shapley, K. (2007). Reviewing the evidence on how teacher professional development affects student achievement. Issues \& Answers Report, REL 2007No. 033. https://ies.ed.gov/ncee/edlabs/regions/southwest/pdf/REL_2007033_sum.pdf. Zugegriffen: 8. März 2017. 